\title{
Topology and Shape Optimization of Ultrathin Composite Self-Deployable Shell Structures with Cutouts
}

\author{
Serena Ferraro* and Sergio Pellegrino \\ California Institute of Technology, Pasadena, California 91125
}

https://doi.org/10.2514/1.J059550

\begin{abstract}
This paper presents two methods to design cutouts that allow damage-free folding of the stiffest possible composite self-deployable thin shell structures of complex shapes. The first method uses level-set functions that define a general number of cutouts. The second method uses a spline representation of the contour of a single cutout and optimizes its shape. Material failure detection is implemented in the solution. Both methods are applied to the design of deployable thin shells forming $90^{\circ}$ joints, and multiple viable solutions are obtained. Experiments on the best performing design, a 90-390 $\mu \mathrm{m}$ thick shell made of Astroquartz with a cyanate ester matrix, with five cutouts on each side, are presented to illustrate and validate the proposed approach.
\end{abstract}

\section{Nomenclature}

\begin{tabular}{|c|c|c|}
\hline$F_{x}, F_{y}$ & $=$ & $\begin{array}{l}\text { in-plane failure strengths, combi- } \\
\text { nation of failure coefficients }\end{array}$ \\
\hline$F_{1 c}=F_{2 c}$ & $=$ & compressive strength parameters \\
\hline$F_{1 t}=F_{2 t}$ & $=$ & tensile strength parameters \\
\hline$F_{3}, F_{4}, F_{6}$ & $=$ & $\begin{array}{l}\text { in-plane shear, bending, and } \\
\text { twisting strength parameters }\end{array}$ \\
\hline$F I_{1}$ & $=$ & in-plane failure index \\
\hline $\mathrm{FI}_{2}$ & & bending failure index \\
\hline $\mathrm{FI}_{3}$ & & $\begin{array}{l}\text { coupled in-plane and bending } \\
\text { failure index }\end{array}$ \\
\hline$f_{i}, f_{i j}$ & & $\begin{array}{l}\text { coefficients, combination } \\
\text { ngth parameters }\end{array}$ \\
\hline$K_{B}$ & & $\begin{array}{l}\text { deployed bending stiffness of } \\
\text { shell }\end{array}$ \\
\hline$M_{x}, M_{y}, M_{x y}$ & $=$ & $\begin{array}{l}\text { bending and twisting moment } \\
\text { resultants for a flat plate }\end{array}$ \\
\hline$N_{x}, N_{y}, N_{x y}$ & $=$ & $\begin{array}{l}\text { in-plane and shear stress resul- } \\
\text { tants for a flat plate }\end{array}$ \\
\hline$R P_{\text {center }}, R P_{\text {left }}, R P_{\text {right }}$ & $=$ & $\begin{array}{l}\text { Reference points for boundary } \\
\text { conditions }\end{array}$ \\
\hline$U_{x}, U_{y}, U_{z}$ & $=$ & $\begin{array}{l}\text { displacements in Cartesian coor- } \\
\text { dinate system }\end{array}$ \\
\hline$\Delta M_{0}$ & & Initial bending moment \\
\hline & & initial folding angle \\
\hline$\theta_{x-R P_{\text {center }}}, \theta_{x-R P_{\text {left }}}, \theta_{x}$ & & $\begin{array}{l}\text { folding angles in Cartesian coor- } \\
\text { dinate system }\end{array}$ \\
\hline
\end{tabular}

\section{Introduction}

$\mathbf{U}$ UTRATHIN composite shell structures, where a shell is defined as ultrathin if the wall thickness is smaller than $400 \mu \mathrm{m}$, provide a versatile low-cost solution for many kinds of deployable structures. The introduction of small cutouts enables damage-free elastic folding that, combined with reverse snapthrough that provides self-locking into the deployed configuration,

Presented as Paper 2019-1524 at the AIAA Scitech 2019 Forum, San Diego, CA, January 7-11, 2019; received 26 February 2020; revision received 6 February 2021; accepted for publication 1 March 2021; published online 10 May 2021. Copyright (ङ 2021 by Serena Ferraro and Sergio Pellegrino. Published by the American Institute of Aeronautics and Astronautics, Inc., with permission. All requests for copying and permission to reprint should be submitted to CCC at www.copyright.com; employ the eISSN 1533-385X to initiate your request. See also AIAA Rights and Permissions www.aiaa. org/randp.

*Currently NASA Jet Propulsion Laboratory, 4800 Oak Grove Drive; serena.ferraro@jpl.nasa.gov.

${ }^{\dagger}$ Joyce and Kent Kresa Professor of Aerospace and Civil Engineering, Graduate Aerospace Laboratories, 1200 East California Boulevard, Mail Code 105-05; sergiop@caltech.edu. Fellow AIAA. can result in stiff, lightweight, and remarkably simple-looking structures. However, only a relatively narrow range of structural configurations have been considered so far, mostly straight booms with parallel dogbone cutouts $[\underline{1}, 2]$, and engineering intuition has played a significant role in the identification of suitable configurations for the cutouts. The general aim of this paper is applying this folding technique to more general situations.

The paper considers the problem of folding a shell with a complex geometry by introducing cutouts, and deploys different tools from the literature on structural optimization of thin shells to demonstrate and experimentally validate the automatic design of cutouts. Both single and multiple cutouts are included in the design space that is investigated, and it is found that the best performing solutions include both types.

Previous studies have optimized the length of cutouts in the edge stiffeners of deployable reflectors [ $\underline{3}$ ] as well as the length, width, and end hole diameter of slots in composite tape-spring hinges [4]. There is rich literature on structural topology optimization, which includes widely used density-based methods, first formalized by Bendsøe and Kikuchi [5], and level-set methods, introduced by Osher and Sethian [6]. A comprehensive review of these methods was presented by Sigmund and Maute [7]. A study by Ye et al. [8] used a level-set method that allows the formation of cutouts to optimize shell structures, but without including geometric or material nonlinearities. Level-set topology optimization methods were also used by Maute et al. [9] to optimize 3D printed composites, and by Geiss et al. [10], for shell structures undergoing large deformations. This existing literature is very relevant to the problem to hand, but it should also be noted that the minimization of structural compliance by optimizing the voids (cutouts) and subject to stress constraints in the folded configuration of a deployable structure poses several unique challenges. The approach chosen in the present paper is a level-set method, in order to allow consideration of a wide range of cutout designs while also keeping the computational effort manageable. An implementation of a density-based method for the cutout optimization of deployable booms can be found in [11].

A specific structure consisting of two cylinders forming a $90^{\circ}$ corner joint, as shown in Fig. 1, is studied in this paper. The diameter of each cylinder is $32 \mathrm{~mm}$, and the distance from the end of a cylinder to the inner intersection is $200 \mathrm{~mm}$. The structure is made of plainweave 525 Astroquartz ${ }^{\circledR}$ II fabric (quartz fiber) [12], pre-impregnated with PMT-F6 cyanate ester resin [13], and the thickness ranges between 90 and $390 \mu \mathrm{m}$. The most highly deformed region, at the intersection between the two cylinders and shown in red in Fig. 1, consists of a two-ply laminate $\left[45_{\mathrm{pw}}\right]_{2}$, where the $0^{\circ}$ direction corresponds to the axis of each cylinder. The thinnest laminate was used in this region to minimize bending related effects. Thicker laminates with up to six plies were used in the rest of the structure.

The aim of the study is to transform this structure into a deployable joint that can be folded without damage. A folding angle of $45^{\circ}$ 


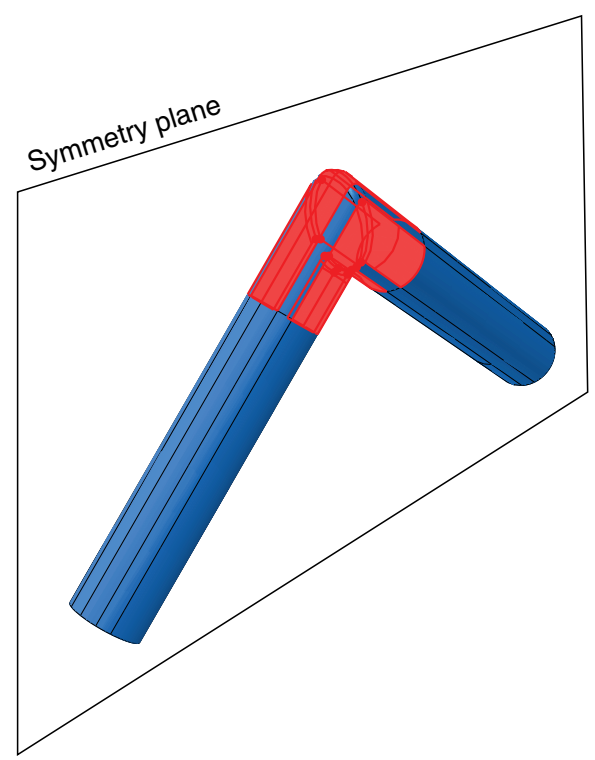

Fig. 1 Thin shell self-deployable joint with two-ply region shown in red.

was chosen, to shorten the time required to carry out geometrically nonlinear finite element simulations and hence to allow fast numerical simulations for the optimization studies. Nonconvergence of iterative finite element solvers is an issue when implementing large displacement simulations of deployable thin shells. Previous authors have addressed this issue by running explicit simulations with very small increments to obtain accurate results $[2,14-16]$ and benchmark problems that compare the implicit and explicit solvers of the Sierra Solid Mechanics code have been proposed [17,18]. Generally, the drawback of choosing an explicit solver is longer simulation times, not a viable option for optimization studies, and therefore an implicit solver was adopted for the present study. The chosen folding angle allowed the study to capture the geometrically nonlinear effects associated with the folding of a corner joint, but avoided the effects of self-contact that would develop for larger folding angles. These effects are also of practical interest for the design of deployable shell structures and will be a topic of future research.

The specific objective of the study is to identify designs for the cutouts in this structure that allow it to withstand folding through $45^{\circ}$ without any damage. The compliance of the resulting structures in the fully deployed configuration is to be minimized.

It should be noted that the shell has a vertical plane of mirror symmetry, shown in Fig. 1 , and all of the cutouts considered in this study maintain this symmetry. Therefore, only the cutouts on one side of the shell will be explicitly discussed and a second set on the other side is assumed.

Two analytical tools were used to carry out this work. First, a finite element software was used to compute the stiffness of each candidate structural design in the deployed configuration and to carry out folding simulations for each design, in order to determine the stress distribution in the folded configuration. Second, a material failure criterion for the two-ply quartz laminate was implemented. These tools are presented in Sec. II. Section III presents two types of designs: the first is based on the simple approach of placing cutouts in the regions of the shell with the highest values of the failure indices in the folded configuration, and the second uses cutouts inspired by previous deployable boom designs, without any formal optimization. Section IV introduces the problem of optimizing the number, location, and shape of cutouts in a shell to allow folding without damage while maximizing the deployed stiffness. Section IV.A presents an optimization approach that uses level-set functions. The best performing design obtained from this approach was implemented, built, and tested. Section IV.B presents the shape optimization of a single cutout. Section $\underline{\mathrm{V}}$ summarizes the outcomes of the study and compares the designs obtained. Section VI concludes the paper.

\section{Analytical Methods}

Finite element simulations were used to analyze the deformation of trial designs of the deployable joint. A failure criterion for two-ply plain-weave laminates was implemented to numerically detect the onset of failure in the most highly stressed region of the joint.

Each simulation computed the bending stiffness of the joint in the initial configuration and the values of the failure indices at each step of the folding process.

\section{A. Finite Element Simulations and Model Parameters}

Geometrically nonlinear finite element simulations were carried out with the commercial software Abaqus 2017. After testing different types of shell elements (S3, S4, and S4R), the S4R elements were chosen because these reduced integration elements run faster and provide results in agreement with experimental results.

The composite layup was not defined explicitly. Instead, the laminate stiffness matrix was defined using the feature "general section properties" in Abaqus, which allows to manually input the $\boldsymbol{A B D}$ stiffness matrix of a shell.

Following Soykasap [19], the $\boldsymbol{A} \boldsymbol{B D}$ matrix of the laminates was calculated with the mosaic model, and the correction factor $\alpha$ (defined as the ratio between the values of $D_{11}$ from the mosaic model and the measured values) was applied to all terms of the $\boldsymbol{D}$ matrix to account for the mosaic model's overprediction of the bending and twisting stiffnesses for laminates with small numbers of plies. The value of $\alpha$ for each laminate was measured from four-point bending experiments [11] and is presented in Table 1 . The effect of the crimp angle on the $\bar{A}$ matrix is relatively small and was neglected [19]. As expected from [19], the values of $\alpha$ decrease and tend to 1 as the number of plies increases.

The $\boldsymbol{A}$ matrix for the laminate at the center of the deployable joint, $\left[45_{\mathrm{pw}}\right]_{2}$, was

$$
\boldsymbol{A}_{\left[\mathbf{4 5}_{\mathrm{pw}}\right]_{2}}=\left[\begin{array}{ccc}
3403.2 & 1872.0 & 0 \\
1872.0 & 3403.2 & 0 \\
0 & 0 & 2150.3
\end{array}\right](\mathrm{N} / \mathrm{mm})
$$

and the $\boldsymbol{D}$ matrix, after introducing the correction factor $\alpha$, was

$$
\boldsymbol{D}_{\left[\mathbf{4 5}_{\mathrm{pw}}\right]_{2}}=\left[\begin{array}{ccc}
3.10 & 1.71 & 0 \\
1.71 & 3.10 & 0 \\
0 & 0 & 1.96
\end{array}\right](\mathrm{N} \cdot \mathrm{mm})
$$

The $\boldsymbol{B}$ matrix was zero because the laminate is symmetric.

The boundary conditions applied to the finite element model are shown in Fig. 2. Three massless reference points, $R P 1, R P 2$, and $R P 3$, were defined. Two rectangular patches, shown in red, were coupled to the left and right reference points such that the in-plane rotations of the patches, $\theta_{x}$, match the rotations imposed to the reference points. The sum of the rotations of the left and right reference points was set equal to the rotation of the center one, and was increased from 0 to $45^{\circ}$. With this boundary condition the end reaction moments are set equal. Finally, two nodes at the bottom of the shell, shown by red dots in Fig. 2, were constrained to remain in the $y-z$ plane, and the three translations of the node at the top of the shell were all set to zero.

The simulations were carried out with the Abaqus implicit solver that uses Newton-Raphson iterations to enforce equilibrium between

Table 1 Bending stiffness $D_{11}(\mathrm{~N} \cdot \mathrm{mm})$ and reduction factor $\alpha$ for two- to six-ply laminates

\begin{tabular}{lcccc}
\hline \hline Laminate & Average $D_{11_{\mathrm{aw} w}}$ & Samples & Standard deviation & $\alpha$ \\
\hline$\left[45_{\mathrm{pw}}\right]_{2}$ & 3.1 & 3 & 0.26 & 2.34 \\
{$\left[45_{\mathrm{pw}} / 0_{\mathrm{pw}} / 45_{\mathrm{pw}}\right]$} & 10.0 & 5 & 0.34 & 2.19 \\
{$\left[45_{\mathrm{pw}}\right]_{4}$} & 23.0 & 5 & 1.10 & 1.69 \\
{$\left[45_{\mathrm{pw}} / 45_{\mathrm{pw}} / 0_{\mathrm{pw}}\right]_{s}$} & 69.8 & 5 & 1.30 & 1.30 \\
\hline \hline
\end{tabular}




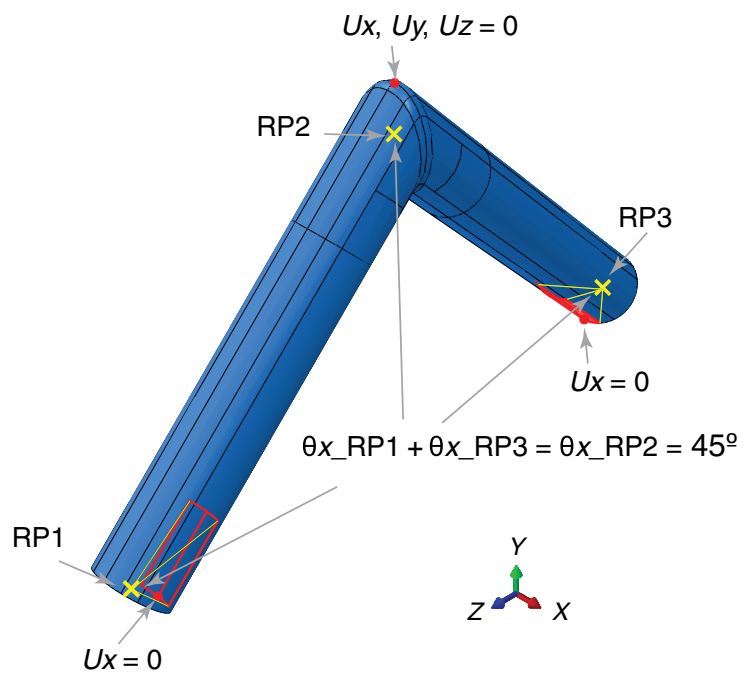

Fig. 2 Finite element model and boundary conditions.

the internal forces and the external loads. Convergence settings based on "half-increment residual tolerance" were used. This tolerance represents the equilibrium residual error (out-of-balance forces) halfway through a time increment. If the half-increment residual is small, it indicates that the accuracy of the solution is high and that the time step can be increased; conversely, if the half-increment residual is large, the time step used in the solution should be reduced. The Abaqus default half-increment residual tolerance, which is set at $10^{-3}$ times the time average force and moment values, was used.

\section{B. Failure Criterion}

Failure predictions during folding were made using the laminate failure criterion by [20]. This criterion, developed for two-ply plainweave carbon fiber composites, is applicable to laminates with fibers of any material and with an even number of plain-weave plies. Hence, it can be applied to the laminate at the center of the deployable joint.

The criterion uses three nondimensional failure indices to capture in-plane, bending, and coupled in-plane and bending failures, defined as follows:

$$
\begin{gathered}
F I_{1}=f_{1}\left(N_{x}+N_{y}\right)+f_{11}\left(N_{x}^{2}+N_{y}^{2}\right)+f_{12} N_{x} N_{y} \\
+f_{33} N_{x y}^{2}<1 \\
F I_{2}=f_{44} \times \max \left(M_{x}^{2}, M_{y}^{2}\right)+f_{66} M_{x y}^{2}<1 \\
F I_{3}=\max \left(\frac{N_{x}}{F_{x}}, \frac{N_{y}}{F_{y}}\right)+\frac{\max \left(\left|M_{x}\right|,\left|M_{y}\right|\right)}{F_{4}}<1
\end{gathered}
$$

where the failure coefficients $f_{i}$ and $f_{i j}$ are given by

$$
\begin{gathered}
f_{1}=f_{2}=\frac{1}{F_{1 t}}-\frac{1}{F_{1 c}} \\
f_{11}=f_{22}=\frac{1}{F_{1 t} F_{1 c}} \\
f_{12}=-\frac{f_{11}}{2} \\
f_{33}=\frac{1}{F_{3}^{2}} \\
f_{44}=f_{55}=\frac{1}{F_{4}^{2}}
\end{gathered}
$$

$$
f_{66}=\frac{1}{F_{6}^{2}}
$$

and

$$
\begin{aligned}
& F_{x}=\frac{-\left(f_{1}+f_{12} N_{y}\right) \pm \sqrt{\left(f_{1}+f_{12} N_{y}\right)^{2}-4 f_{11}\left(f_{1} N_{y}+f_{11} N_{y}^{2}+f_{33} N_{x y}^{2}-1\right)}}{2 f_{11}} \\
& F_{y}=\frac{-\left(f_{1}+f_{12} N_{x}\right) \pm \sqrt{\left(f_{1}+f_{12} N_{x}\right)^{2}-4 f_{11}\left(f_{1} N_{x}+f_{11} N_{x}^{2}+f_{33} N_{x y}^{2}-1\right)}}{2 f_{11}}
\end{aligned}
$$

The terms $F_{i}$ represent directly measured or calculated strengths in the tow directions, and the subscripts $t$ and $c$ denote tension and compression, respectively. In a plain-weave laminate $F_{1 t}=F_{2 t}$ and $F_{1 c}=F_{2 c}$.

The tensile strength was measured as the smallest failure stress resultant obtained from tensile tests on plain-weave $\left[0_{\mathrm{pw}}\right]_{2}$ laminates on 15-mm-wide test samples. The compressive strength was calculated using elastoplastic fiber microbuckling theory [21]

$$
F_{1 c}=\frac{G}{1+\left(\phi_{0} / \gamma_{y}\right)} t
$$

where $\phi_{0}$ is the fiber misalignment angle, measured from micrographs of the laminate, $\gamma_{y}$ is the in-plane yield shear strain, and $t$ is the tow thickness. $\gamma_{y}$ was derived from direct measurements of the shear strength $\tau_{y}$, with

$$
\gamma_{y}=\frac{\tau_{y}}{G}
$$

and $G$ is the shear modulus of a composite tow:

$$
G=\frac{G_{m}\left(G_{12 f}+G_{m}+V_{f}\left(G_{12 f}-G_{m}\right)\right)}{G_{12 f}+G_{m}-V_{f}\left(G_{12 f}-G_{m}\right)}
$$

The reason why the compressive strength was not obtained from direct measurements is that it has been shown [22] that compression tests on thin plain-weave composites have a low success rate due to test sample delamination and other spurious failure modes occurring instead of fiber microbuckling. It was found [22] that the prediction method described above estimates the microbuckling failure load with good accuracy. The in-plane shear strength $F_{3}$ was measured from tension tests on $\left[45_{\mathrm{pw}}\right]_{2}$ laminates. The bending strength $F_{4}$ and the twisting strength $F_{6}$ were obtained from the smallest failure moments measured from platen bending tests $[20,23,24]$, respectively, on $\left[0_{\mathrm{pw}}\right]_{2}$ and $\left[45_{\mathrm{pw}}\right]_{2}$ laminates.

Table $\underline{2}$ shows the resulting strength parameters for two-ply laminates.

Initially, the three failure indices in Eqs. (3-5) were calculated at each step of the folding simulations, and it was found that $F I_{1}$ was the largest of the three and the only failure index with values above 1 . Because of the extreme thinness of the shells, $\mathrm{FI}_{2}$ and $\mathrm{FI}_{3}$ tend to be

Table 2 Material strength parameters for two-ply laminates of Astroquartz $^{\circledR}$ fiber and cyanate ester resin

\begin{tabular}{lccc}
\hline \hline Strength parameter & Average value & Samples tested & Standard deviation \\
\hline$F_{1 t}=F_{2 t}[\mathrm{~N} / \mathrm{m}]$ & 76.16 & 5 & 2.83 \\
$F_{1 c}=F_{2 c}[\mathrm{~N} / \mathrm{mm}]$ & 34.50 & - & -- \\
$F_{3}[\mathrm{~N} / \mathrm{mm}]$ & 14.55 & 5 & 0.12 \\
$F_{4}[\mathrm{~N}]$ & 3.26 & 4 & 0.28 \\
$F_{6}[\mathrm{~N}]$ & 1.10 & 4 & 0.06 \\
\hline \hline
\end{tabular}


smaller. Therefore, it was decided to focus on $F I_{1}$ in the optimization study.

\section{Preliminary Designs}

Folding simulations of the shell without any cutouts were used to identify an initial location for a set of two identical cutouts, symmetrically placed on each side of the joint. Figure 3 shows the midplane stress resultants in the longitudinal direction of the two cylinders. The figure shows two peaks, one tensile and one compressive, in a narrow region at the center of the shell.

Based on this initial result, it was conjectured that the cutout topology problem could be solved simply by "drilling out" the most highly stressed part of the shell. Hence, a series of joints with circular cutouts over the stress peak region in Fig. 3 were analyzed. These attempts were all unsuccessful, even when the cutout with an initial diameter of $14 \mathrm{~mm}$ was enlarged.

An example is shown in Fig. 4, which shows a plot of $F I_{1}$ in the two-ply region of the joint. White areas of the plot are regions corresponding to cutouts or parts of the shell that were excluded from the failure analysis. Black areas in Fig. 4 are regions where $F I_{1}<1$ and hence, according to the failure criterion, will not fail. Finally, all the regions plotted with a color other than white or black are predicted to fail. This result is typical of cutouts introduced without a formal analysis: instead of disappearing, the high stress concentrations move to the edges of the new cutouts.

To remove wider areas of the shell, elliptical cutouts were considered. Figure 5 shows a cutout obtained by intersecting two ellipses and symmetrically varying the major and minor axes of each ellipse. Again, the first failure index was greater than one in several parts of the shell; see the regions circled in red in the figure.

Further attempts to find a simple, viable cutout shape were made. The cutout in Fig. 6 was inspired by previous designs for cylindrical booms with slotted hinges $[1,4]$. This cutout shape greatly decreases the extent of the damage but does not fully prevent it. Even wider cutouts were also considered, and finally a design that fully succeeds

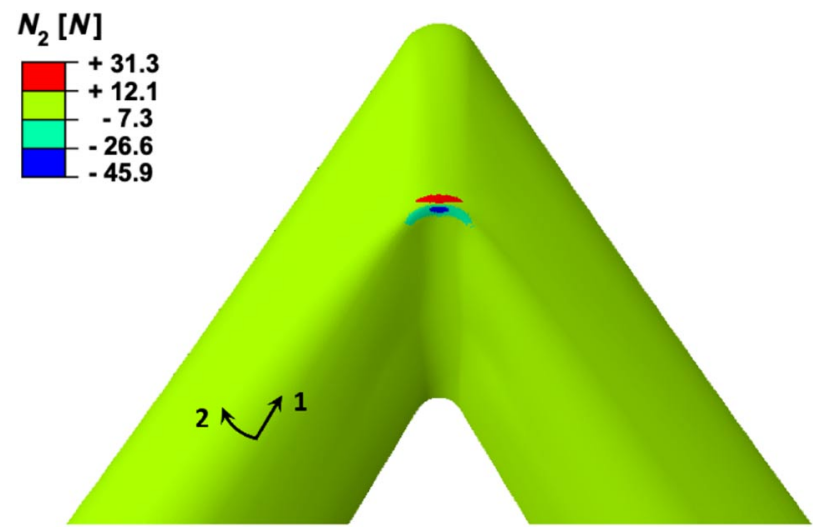

Fig. 3 Midplane stress resultant in two directions for shell without cutouts folded through $45^{\circ}$.

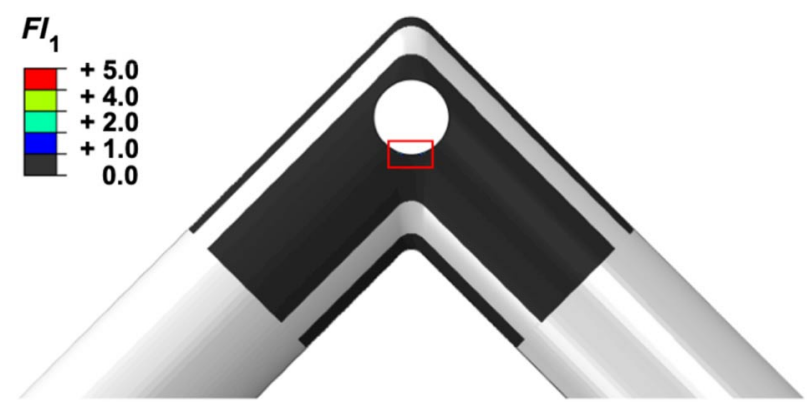

a) Overall view

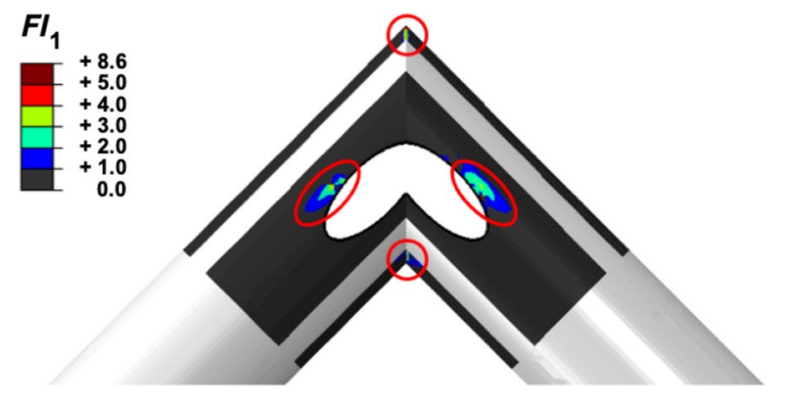

Fig. 5 Contour plot of $F I_{1}$ (mapped on deployed configuration) for joint with sharp corners and cutout consisting of two intersecting ellipses, folded through $45^{\circ}$.

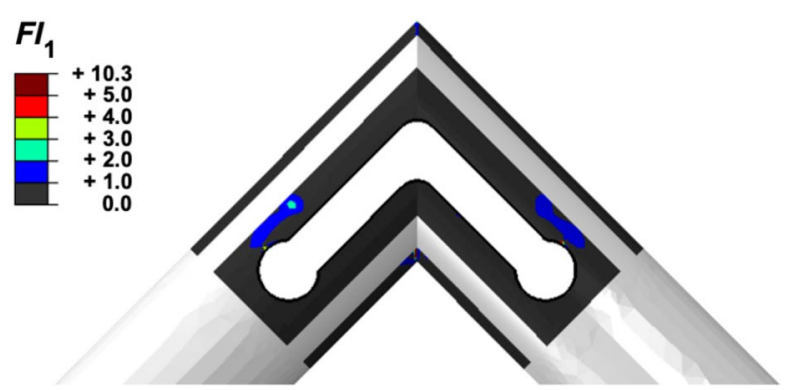

Fig. 6 Contour plot of $F I_{1}$ on shell folded $45^{\circ}$ with cutout inspired by booms with slotted hinges. $F I_{1}$ has been mapped on the deployed shell.

in avoiding any damage is shown in Fig. 7, in which the two-ply region of the joint has been largely removed. This type of cutout changes the folding behavior of the joint, as it allows two localized elastic hinges to form near the ends of the cylinders, and the $90^{\circ}$ corner tape spring at the top opens out when the joint is folded. A problem with this approach is that, while being successful in resolving the issue of material damage, it also greatly reduces the deployed stiffness of the shell.

The main conclusion, after the preliminary design attempts presented in this section, was that the simple approach of designing the cutouts by removing the most highly stressed material does not work well. A more rigorous formulation of the problem is needed, together with a solution method that is compatible with the adopted formulation.

\section{Optimization Methods}

The problem of finding the shape, number, and position of cutouts to optimize the stiffness and satisfy the failure constraints of a deployable shell forming a $90^{\circ}$ joint can be defined as follows:

$$
\begin{array}{ll}
\min _{x_{1} \ldots x_{n}} & :-K_{B} \\
\text { subject to } & : \alpha_{i}<x_{i}<\beta_{i}, \\
& : \max \left(F I_{1}\right)<1
\end{array} \quad i=1,2,3, \ldots, n
$$

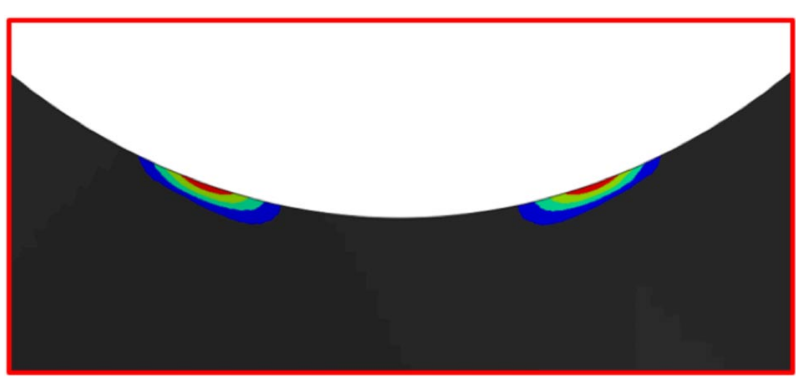

b) Close-up

Fig. $4 F I_{1}$ for shell folded through $45^{\circ}$ mapped on deployed configuration. 


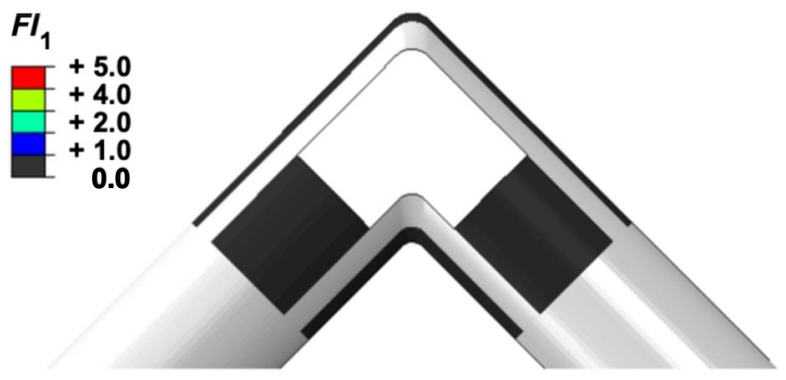

a) Contour plot of $\mathrm{FI}_{1}$ (plotted on deployed configuration)

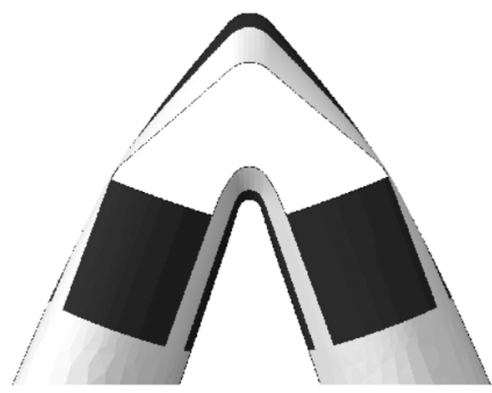

b) Contour plot of $\mathrm{FI}_{1}$ on shell folded through $45^{\circ}$

Fig. 7 Joint with smooth corners and partially removed two-ply region.

where the objective function $K_{B}$ is the bending stiffness of the joint in the deployed configuration and $n$ is the number of design variables. The two sets of inequality constraints specify that the cutouts should remain within prescribed geometric boundaries, $\alpha$ and $\beta$, defined by the geometry of the shell and there should be no damage of the shell during folding, thus constraining the maximum value of the first failure index to be less than one (the second and third failure indices were not included in the optimization, as noted in Sec. II.B).

Because of the interaction between the shape and position of the cutouts as well as the interaction with the constraints, the optimization problem defined in Eq. (11) is likely to be nonconvex. The Appendix provides further details. Hence, a robust solution method has to examine changes in the objective associated with small changes in the design parameters and also the possibility of achieving greater changes in the objective by switching from one cutout to two, etc. Taking a practical viewpoint, both local and global changes of cutout designs need to be considered for a thorough study of the design space.

Several topology optimization methodologies were investigated [11], including a density-based approach (using both SIMP and RAMP interpolation schemes) that was implemented on simpler shell structures, where convexity of the optimization problem could be assumed. This approach has the advantage that no assumption is made regarding the initial position or shape of the cutouts. It is applicable to any structural problem, but the large number of design variables, equal to the number of elements in the finite element discretization of the structure, is a significant limitation if the optimization problem is nonconvex. This is the most likely scenario in the present case, and for this reason a level-set approach was chosen. This approach, presented in the next subsection, can be used to explore general configurations of the cutouts relatively quickly. A second approach, which can be used to optimize the shape of a particular cutout [25], is then presented in the following subsection.

\section{A. Topology Optimization Using Level-Set Functions}

Level-set methods change the topology of a structure by moving the geometric boundaries [26-29]. A scalar level-set function is based on the following definition: if a point within the design domain corresponds to negative values of the function, the point is assigned to the void domain; if it corresponds to positive values of the function, the point belongs to the solid domain; finally, null values of the function describe the boundaries of the structure.

In two dimensions, a closed curve $\Gamma$ is defined by the auxiliary function $\phi$, called the level-set function. $\Gamma$ is defined by $\phi=0$ and the level-set method manipulates $\Gamma$ implicitly (i.e., without an explicit parameterization of the contour of the curve), through the function $\phi$.

This method allows for a broad exploration of the shape, number, and position of the cutouts. The algorithm assigns values to the design variables and then generates new shapes for the cutouts based on these values. Once these shapes are created, a Python script generates an input file for the finite element software, Abaqus 2017, which runs a finite element analysis and returns the values of the first failure index and the overall bending stiffness of the structure.

\section{Method Description}

A basis function, $z=f\left(x_{p}, y_{p}\right)$, is defined on a plane tangent to the shell. The basis function is chosen such that it vanishes at the boundaries of its domain, and hence the geometric constraints of the optimization problem in Eq. (11) are automatically satisfied. A cutting plane, parallel to the $x_{p} y_{p}$ plane, is then introduced, and the intersection of the plane and the basis function defines a set of contour shapes that determine a set of cutouts.

The method consists of four steps, shown in Fig. 8. The first step, Figs. $8 \mathrm{a}$ and $8 \mathrm{~b}$, finds a mapping between the domain on which the basis function is described (in this case a square), and the domain on which the contours of the cutouts are generated (in this case an Lshaped, smooth domain that represents a portion of the projection of the shell). The second step, Fig. $\underline{8 c}$, chooses a basis function and applies the mapping to it. Two basis functions were investigated: a series of cosines squared,

$$
\begin{aligned}
& z=\sum_{h=1}^{N_{h}} \sum_{l=1}^{N_{l}} a_{n}\left[\cos (2 h+1) \frac{\pi x_{p}}{2} \times \cos (2 l+1) \frac{\pi y_{p}}{2}\right]^{2}, \\
& n=1,2,3, \ldots, N_{h} N_{l}
\end{aligned}
$$

and a series of cosines and sines squared,

$$
\begin{aligned}
z & =\sum_{h=1}^{N_{h}} \sum_{l=1}^{N_{l}} a_{n}\left[\left(\cos (2 h+1) \frac{\pi x_{p}}{2}+\sin \left(h \pi x_{p}\right)\right)\right. \\
& \left.\left(\cos (2 l+1) \frac{\pi y_{p}}{2}+\sin \left(l \pi y_{p}\right)\right)\right]^{2}, \quad n=1,2,3, \ldots, N_{h} N_{l}(13)
\end{aligned}
$$

These functions were chosen because they vanish at the boundaries of the chosen domain, which is a square defined by $x_{p}= \pm 1$ and $y_{p}= \pm 1$, and because the number of peaks of these functions can be easily changed by increasing the number of terms in the series.

The third step, Fig. 8d, consists in intersecting the chosen basis function with a plane parallel to the $x_{p} y_{p}$ plane. For a chosen basis function, the only design variables used to define the problem and carry out the optimization analysis are the $z$ coordinate of the cutting plane, $z=c$, and the numbers of terms in the series, $N_{h}$ and $N_{l}$, which define the number of peaks of the function. Together, the parameters $c, N_{h}, N_{l}$ define the number of cutouts. Note that the cutting plane need not be parallel to the $x_{p} y_{p}$ plane, as two slopes could be included as additional design variables, to generate a wider range of shapes.

For example, the basis function in Eq. (12) with $N_{h}=N_{l}=1$ with the planes, $z=c_{1}$ and $z=c_{2}$, gives the cutouts shown in Fig. 9 .

\section{Results}

Because the number of design variables is small, no particular optimization algorithm was used. Instead, the entire design space was evaluated. Three cutout designs generated using level-set optimization are shown in Fig. 10. The first design, Fig. 10a, was obtained from Eq. (12) for the choice $N_{h}=0$ and $N_{l}=\overline{2}$, and hence the shapes of the cutouts are symmetric. This design provides the highest 


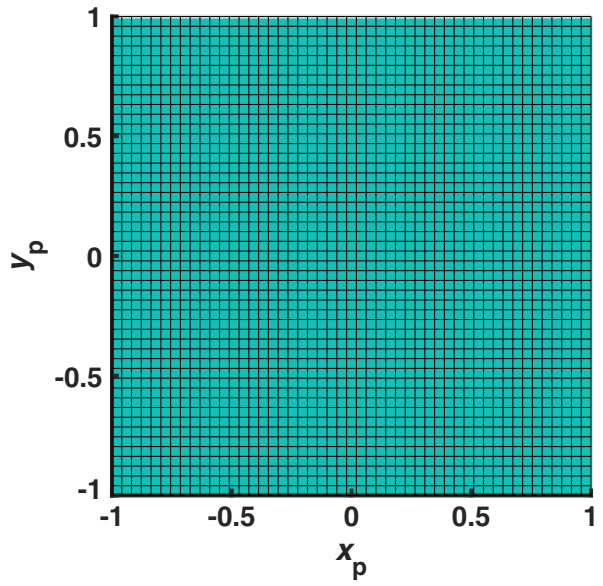

a) Choice of domain for basis function

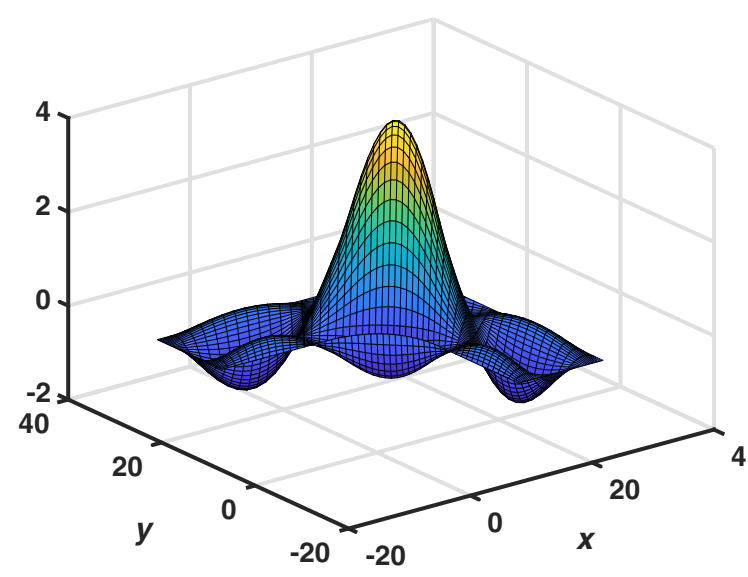

c) Choice of basis function

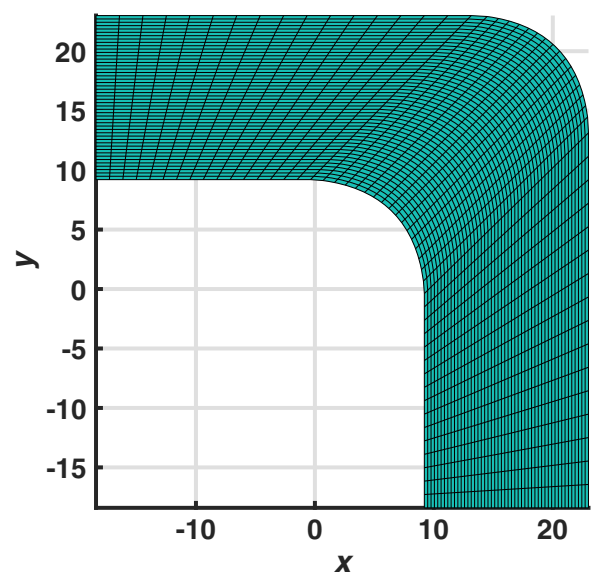

b) Mapping to structural domain

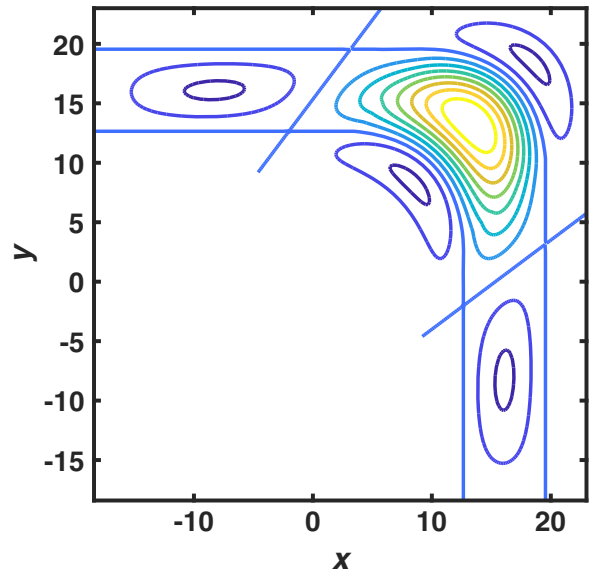

d) Mapping and intersection with plane $z=$ constant

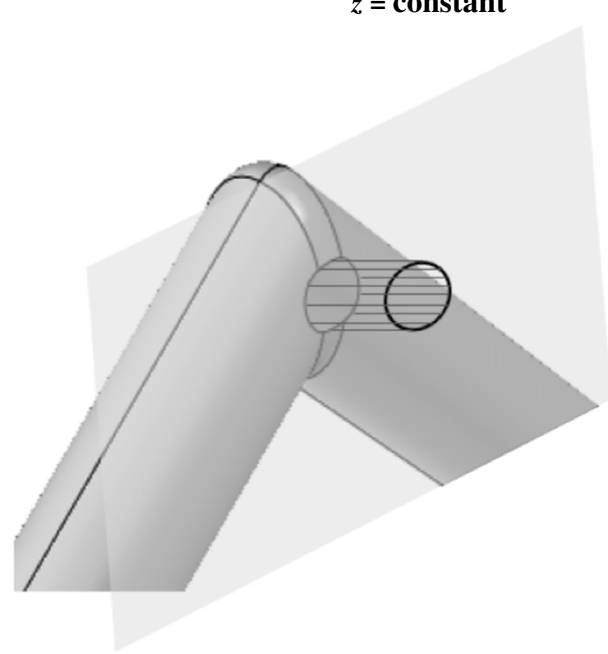

e) Projection of cutout on the shell

Fig. 8 Steps of topology optimization method using level-set functions.

bending stiffness while also satisfying the constraints on the maximum values of the failure index. Figure 10a also shows a plot of $K_{B}$ versus $\max \left(F I_{1}\right)$ for different values of $c$; among the designs with $\max \left(F I_{1}\right)<1$ the stiffest corresponds to the point $(0.85,2078)$. Note that the boomerang-shaped cutouts in this design leave strips of material connecting the right and left parts of the shell, which increases the deployed bending stiffness of the joint.

The second design, Fig. 10b, was also obtained using Eq. (12), for the choice $N_{h}=1$ and $\overline{N_{l}}=2$. The continuous cutout at the center of the joint removes areas of localized stresses while thin slits on the sides help to decrease the highest stresses when the joint is folded.

Finally, the third design, Fig. 10c, was obtained using Eq. (13), which results in a nonsymmetric cutout. The shape in Fig. 10c was obtained by choosing $N_{h}=2$ and $N_{l}=0$. Despite being the best result obtained using a nonsymmetric basis function, this larger cutout leads to a joint with a much lower bending stiffness when compared with the symmetric cutout designs. 


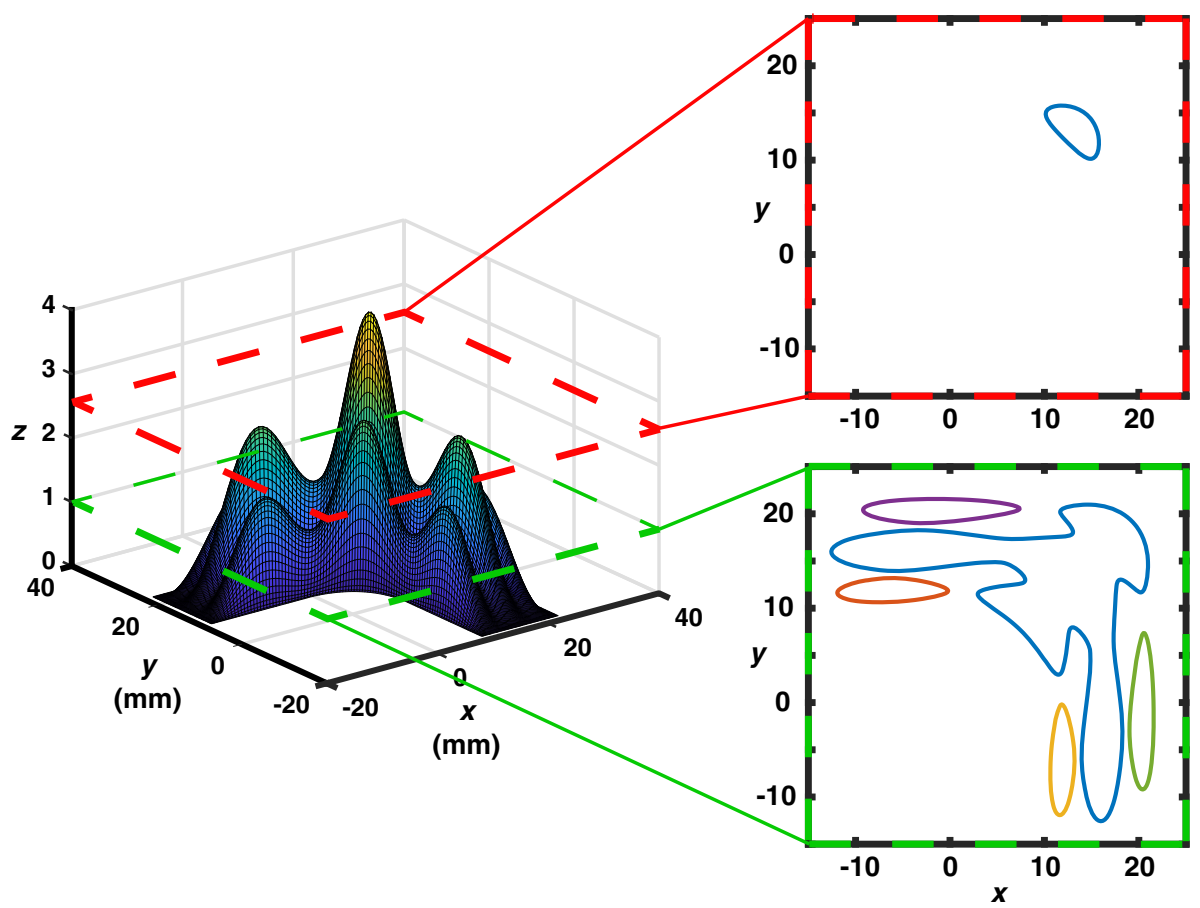

Fig. 9 Cutout shapes obtained by intersecting the basis function in Eq. (12) with $N_{h}=N_{l}=1$, with planes $z=1$ and $z=2.6$.

\section{Experimental Verification}

To experimentally verify the best result from the level-set optimization method, a self-deployable joint was built using a silicon molding technique [30]. The cutouts in Fig. 10a were laser-cut after demolding the joint.

A quasi-static folding experiment was conducted using the setup shown in Fig. 11a. Two $35 \mathrm{~mm} \times 15 \mathrm{~mm}$ curved clamps conforming to the curvature of the cylindrical shells constrained small regions of the shell, thus leaving the end cross sections free to ovalize. The joint was mounted on the test setup through brackets attached to gearboxes, connected to strain gauges and to a data acquisition system, in an initially stress free configuration. One of the gearboxes was mounted on ball bearings, to allow it to slide toward the other gearbox. The experiment consisted in manually rotating both ends of the joint by equal amounts, through the gearboxes. First, the sliding bracket was rotated by a small angle $\left(1-3^{\circ}\right)$, and the corresponding reaction moment was recorded. Second, the fixed bracket was rotated until the reaction moment equaled the moment recorded on the sliding bracket. This process was repeated until the joint had been folded through $45^{\circ}$. At the end of the test, the moment-rotation profile shown in Fig. $11 \mathrm{~b}$ was obtained by plotting the folding angle and the corresponding moment at each step.

The moment-rotation profile resulting from the numerical simulations described in Sec. II.A, in which the boundary conditions matched the experiment, has been superposed to the experimental results. This comparison shows a very good agreement for the first $27^{\circ}$ of folding, but there is a deviation of up to $12 \%$ for the last $18^{\circ}$. The reason for this deviation becomes clear when one looks closely at the deformed shape of the shell near the cutouts for a fold angle greater than $27^{\circ}$, as shown in Fig. 12. Comparing the actual deformed shapes of the five strips to the finite element simulation, it can be noticed that four of the five shapes are similar, but the actual shape of the central strip (identified by red arrows) is curved inward, whereas in the simulation it is curved outward. The curvature reversal of this strip is associated with a snapthrough instability along the main equilibrium path of the shell and is triggered by an unmodeled shape imperfection of the shell. This reversal of the strip curvature corresponds to a softening of the moment-rotation response of the shell. Examples of this type of behavior have been studied by Royer and Pellegrino [31].

Two results stand out from this experiment. First, the initial slope of the moment-rotation curve from the simulation matches the experimental measurements. This slope measures the stiffness of the deployed joint:

$$
K_{B}=\frac{\Delta M_{0}}{\Delta \theta_{0}}
$$

where $\Delta M_{0}$ is the increment in bending moment measured on the tangent to the moment-rotation curve at the initial point $(M=0$, $\theta=0$ ), and $\Delta \theta_{0}$ is the corresponding increment in folding angle, measured in radians). Second, no damage was observed after folding the joint three times, and the measured bending stiffness in the deployed configuration did not change after the shell had been folded.

\section{B. Shape Optimization of Single Cutout}

A contour was defined on a plane tangent to the side of the shell, using a set of eight control points and a spline fit between them. The tangent plane provides a convenient Cartesian coordinate system to describe the control points. The contour was then projected onto the shell. The coordinates of each control point, $x_{i}, y_{i}$, in the tangent plane were chosen as design variables for the minimization problem. A visualization of the design space is provided in Fig. 13.

The geometric constraints in Eq. (11) were initially defined such that the control points remain within the red contour, Fig. 13, corresponding to the large cutout in Fig. 7. Constraining the control points to fit within this contour ensures that the cutout remains within the physical boundaries of the shell.

Since the control points can be placed anywhere within the red contour, and the position of each point is independent of the other points, two issues emerge regarding these geometric constraints. The first issue is that the red contour is nonconvex, and hence the spline connecting the control points can escape the contour. To prevent this, the control points were originally defined on a square domain, Fig. 14a, with Cartesian coordinates $x_{p}, y_{p}$ :

$$
x_{p} \in[-1,1], \quad y_{p} \in[-1,1]
$$

The points were then mapped to an L-shaped domain, Fig. 14b, with Cartesian coordinates $x, y$ via the following mapping, which uses polynomial functions whose coefficients were found by substituting the points that lie on the boundary of the square and L-shaped domains 


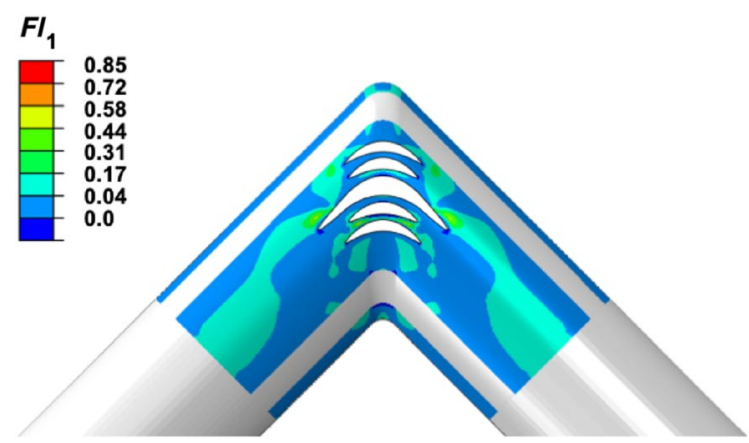

a) Eq. 12 and $N_{\mathrm{h}}=0, N_{\mathrm{l}}=2 . \mathrm{c}=[0.8,1.65]$ with step size 0.05
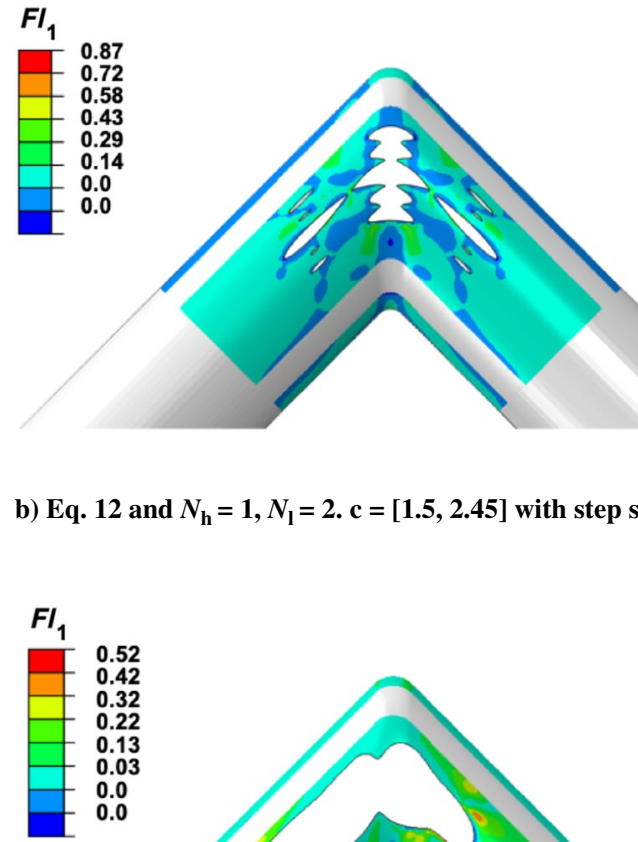

b) Eq. 12 and $N_{\mathrm{h}}=1, N_{\mathrm{l}}=2 . \mathrm{c}=[1.5,2.45]$ with step size 0.05

b) Eq. 13 and $N_{\mathrm{h}}=2, N_{\mathrm{l}}=0 . \mathrm{c}=[0.01,3.01]$ with step size 0.1
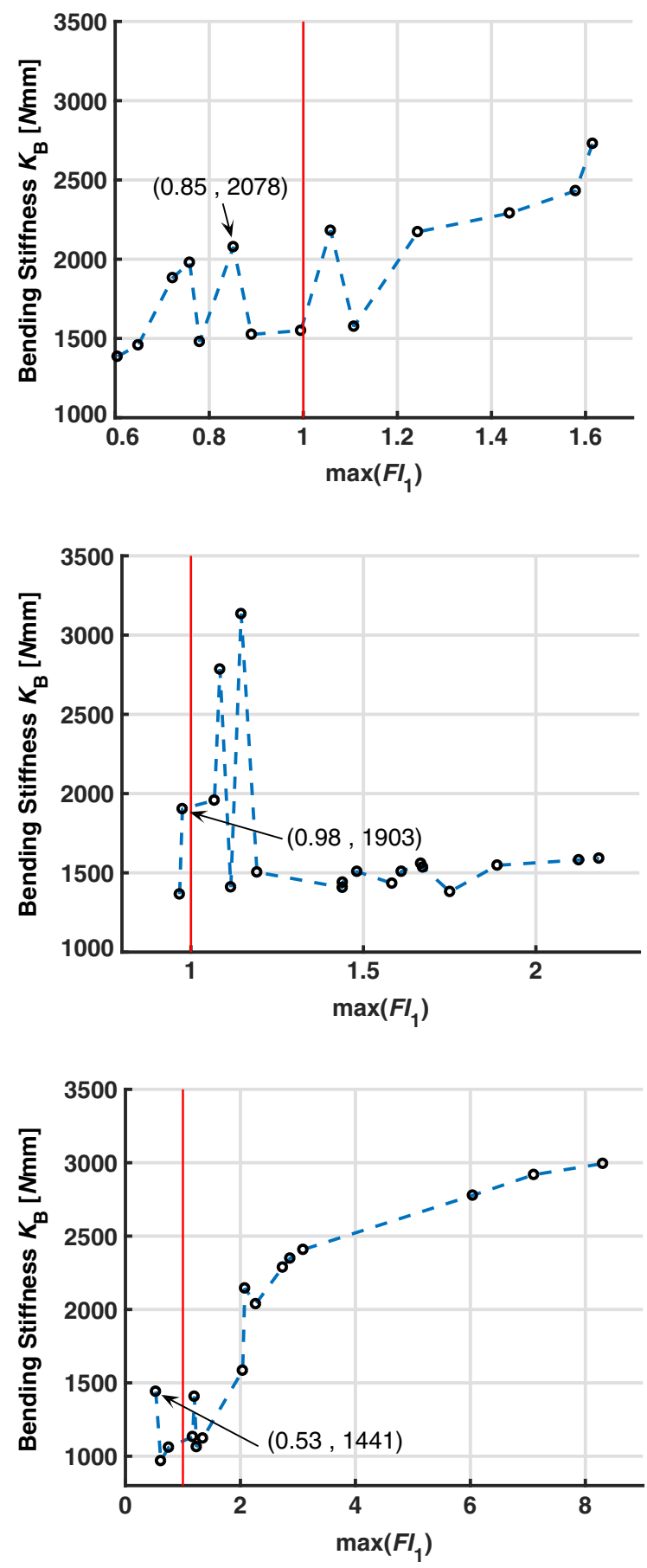

Fig. 10 Results from level-set method.

for $x_{p}<-1 / 3$,

$$
\begin{gathered}
x=-12+8 y_{p} \\
y=-15-21 x_{p}+12 y_{p}+12 x_{p} y_{p}
\end{gathered}
$$

for $x_{p}>1 / 3$,

$$
\begin{gathered}
x=-15+21 x_{p}+12 y_{p}-12 x_{p} y_{p} \\
y=-12+8 y_{p}
\end{gathered}
$$

and for $-1.3 \leq x_{p} \leq 1 / 3$,

$$
\begin{aligned}
& x=-10.83+6 x_{p}+8 y_{p}+7.46 x_{p}^{2} \\
& y=-10.83-6 x_{p}+8 y_{p}+7.46 x_{p}^{2}
\end{aligned}
$$

The second issue, how to avoid self-intersection of the spline, was addressed by constraining each control point to remain within a subdomain of the square domain, displayed with dotted lines in Fig. 14a.

\section{Mesh Convergence Studies}

The sensitivity to the mesh size of the objective function in Eq. (11) and of the associated constraints was studied across a range of cutout shapes. The circular cutout in Fig. 15a was used as the initial cutout

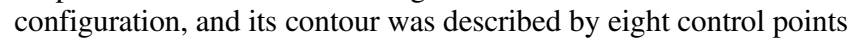
connected by a spline. One coordinate, $x$, of a single control point was 


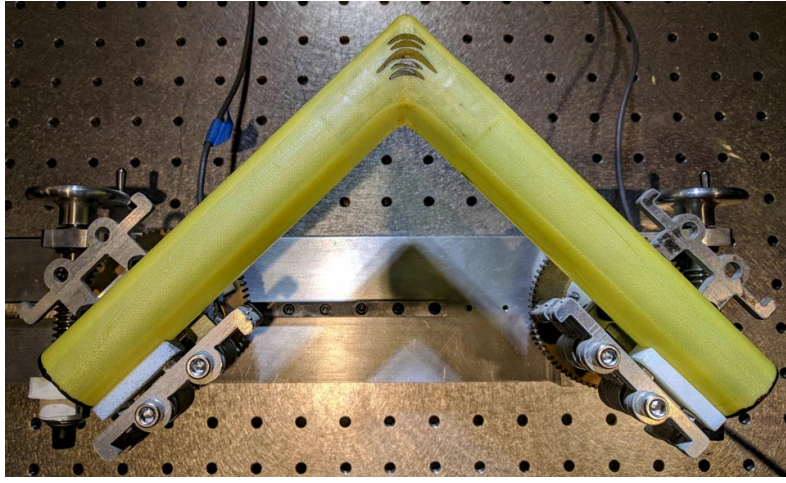

a) Test setup

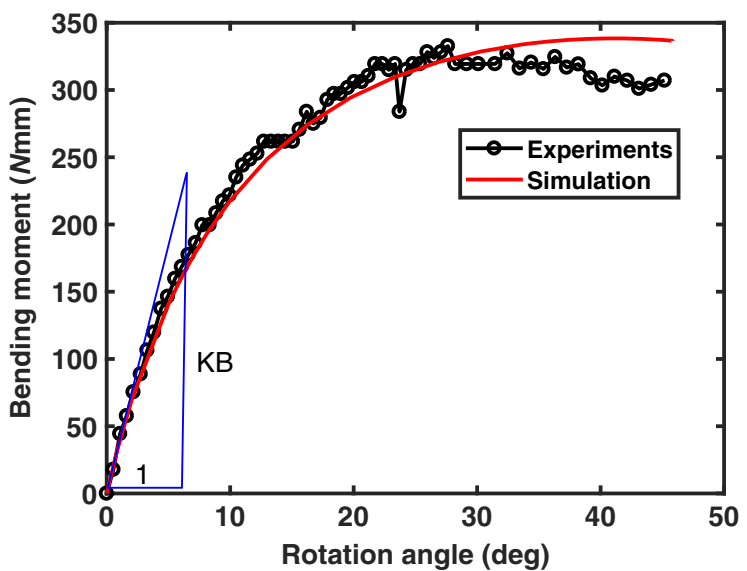

b) Comparison of test and simulation results

Fig. 11 Folding test.

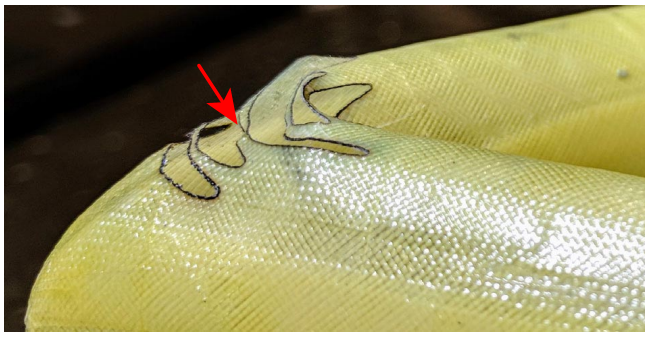

a)

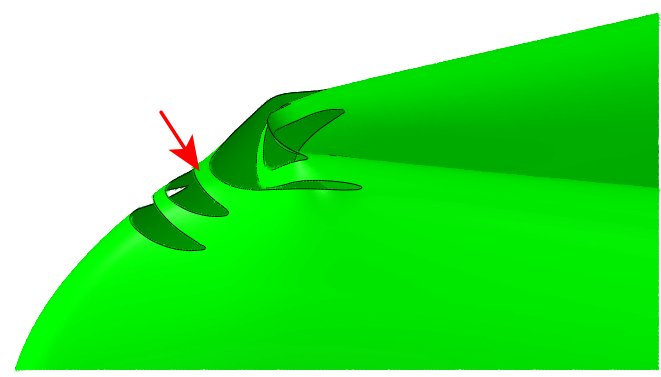

b)

Fig. 12 Deformed shape of the shell near the cutout: a) experiment and b) simulation.

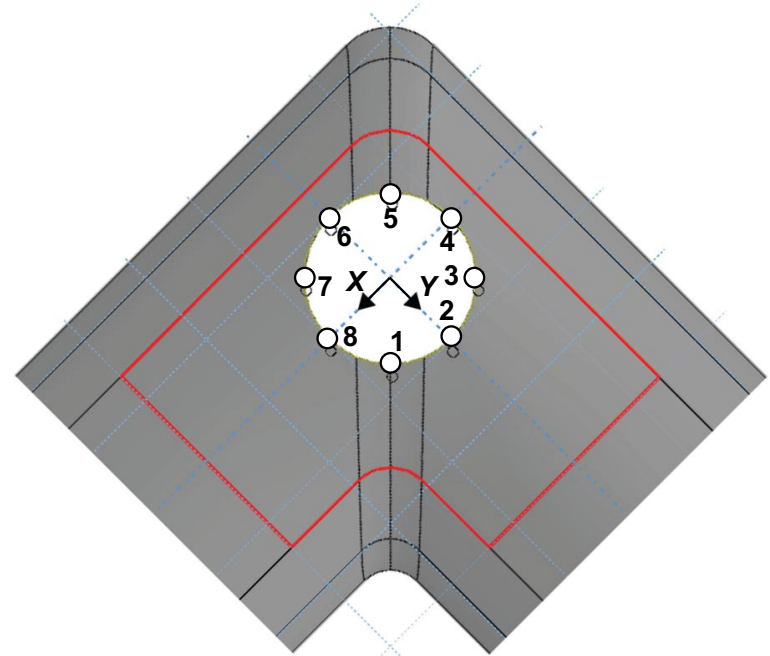

Fig. 13 Design space for shape optimization of single cutout.

increased from $4.95 \mathrm{~mm}$, Fig. 15a, to $12.7 \mathrm{~mm}$, Fig. $15 \mathrm{~b}$, whereas the other control points were held fixed. For each value of $x$, a geometrically nonlinear finite element simulation of folding was carried out.

The values of the deployed joint stiffness and of the maximum failure index $F I_{1}$ that were obtained from this analysis are plotted in Figs. $15 \mathrm{c}$ and $15 \mathrm{~d}$, respectively, for four mesh sizes. In these figures, the indicated mesh size is the average size of the S4R elements near the cutout.

One of the four meshes, with average element size of $0.125 \mathrm{~mm}$ near the cutout, is shown in Fig. 15e. The size of the elements far away from the cutout is determined by the largest distance allowed between consecutive nodes such that a smooth transition region between larger and smaller elements can be generated. The aspect ratio of the elements was set to be no larger than 3 .

Figure $15 \mathrm{c}$ shows that the bending stiffness $K_{B}$ is insensitive to the mesh size, as the stiffnesses computed with the four different mesh sizes are practically identical across the full range of $x$. Figure $15 \mathrm{~d}$ shows significant variations in the maximum value of $F I_{1}$ when the mesh size near the cutout is decreased from 0.5 to $0.125 \mathrm{~mm}$; however, there is practically no difference when the mesh is further refined to $0.0625 \mathrm{~mm}$ (the yellow and purple curves in the figure differ by $<1 \%$ for all values of $x$ ). It is therefore concluded that accurate estimates of both $K_{B}$ and $\max \left(F I_{1}\right)$ are obtained for a mesh size of $0.125 \mathrm{~mm}$.

\section{Cutout Optimization}

After completing these initial studies, a search for a global minimum was carried out with a global search method. The global optimization was performed with the Basin-Hopping algorithm [32], a stochastic, two-phase method that combines a global stepping algorithm with local minimization at each step. The algorithm iterates first by performing random perturbations of the design variables, second by performing local optimization, and third by accepting or rejecting new design variables based on an acceptance test. The local minimization was performed with Constrained Optimization BY Linear Approximation (COBYLA) [33], an optimization algorithm for constrained problems that does not use gradient information. The acceptance test used was the Metropolis criterion of standard Monte Carlo algorithms [34], although there are many other possibilities.

During an iteration, a linear approximation of the objective function is solved to obtain a candidate for the optimal solution. The candidate solution is evaluated using the original objective and constraint functions, yielding a new data point in the optimization space. This information is used to improve the linear approximation for the next iteration. When the solution cannot be improved, the step size is reduced and the search is refined. When the step size becomes sufficiently small, the algorithm stops. 


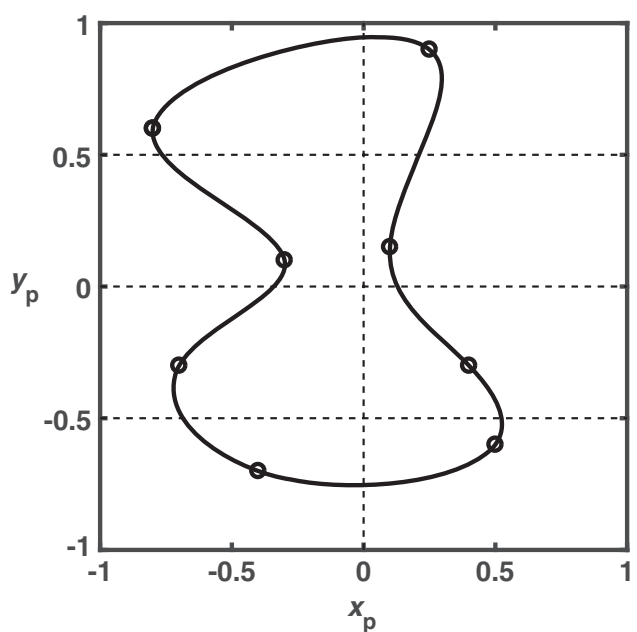

a) Definition of control points and spline on square domain

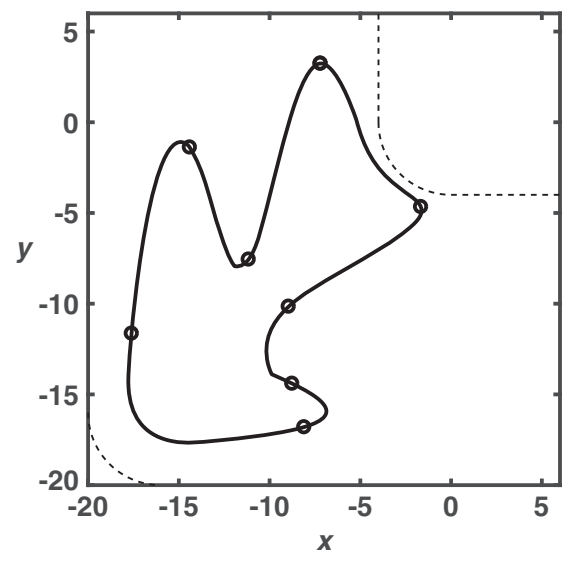

b) Mapping to L-shaped domain

Fig. 14 Geometrical constraints for shape optimization problem.

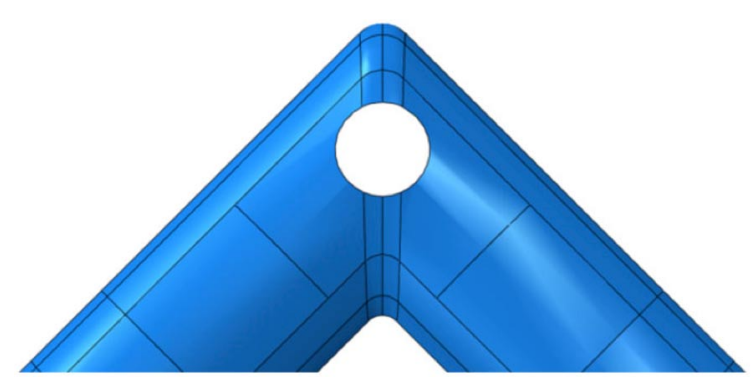

a) Initial guess $x=4.95 \mathrm{~mm}$

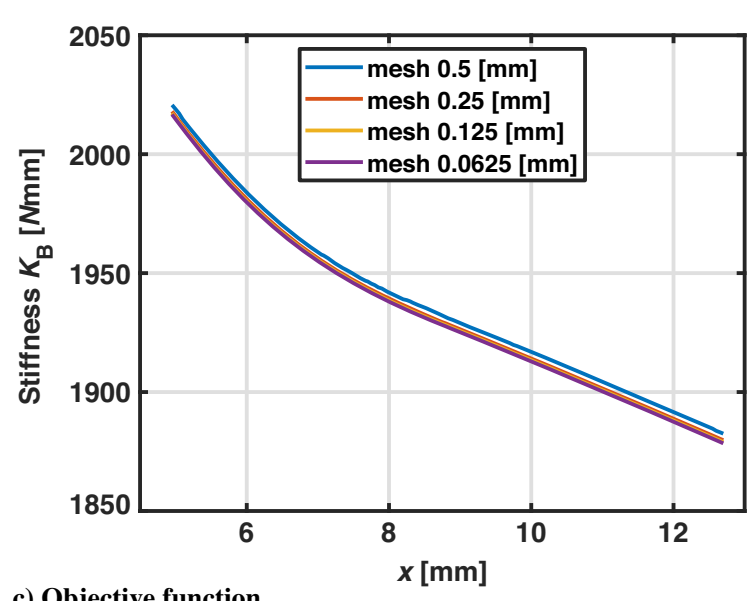

c) Objective function

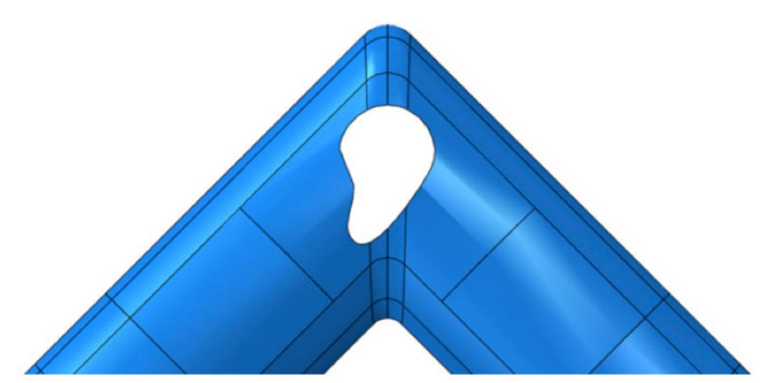

b) Final shape $x=12.7 \mathrm{~mm}$

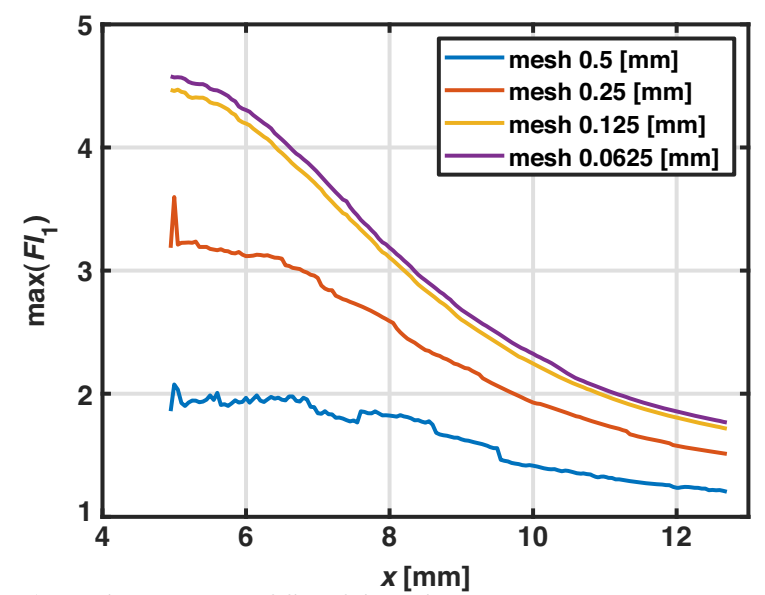

d) Maximum value of first failure index

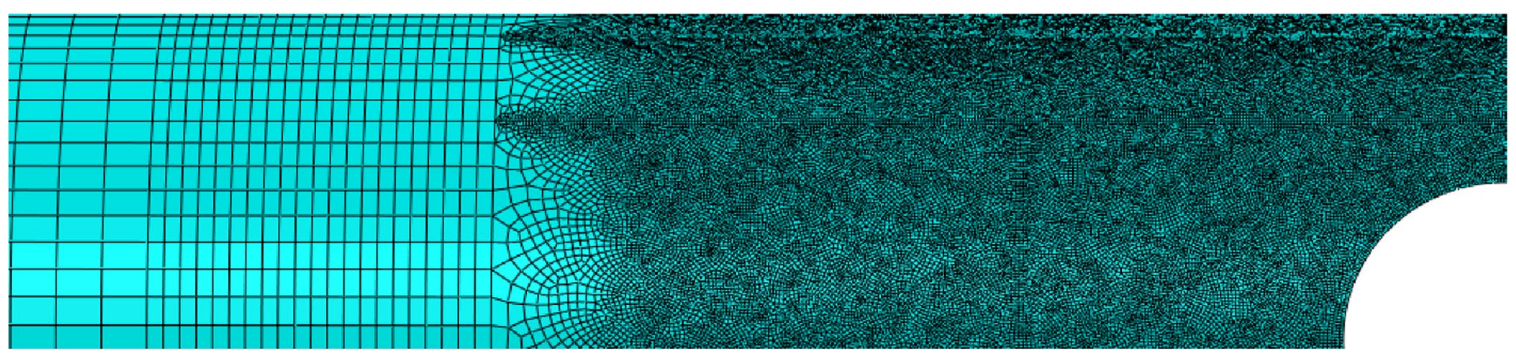

e) Example of finite element mesh

Fig. 15 Mesh convergence study. 
The Basin-Hopping and COBYLA algorithms are available in publicly available libraries for Python scripts [35] and were incorporated within the parametric optimization method. A Python script was used to generate an input file for Abaqus 2017, with the cutout shape defined by specific design variables. The Abaqus software runs a finite element analysis of the prescribed joint design and returns the values of $F I_{1}$ and $K_{B}$. The script evaluates the objective function and calls the Basin-Hopping and COBYLA algorithms to update the design variables. Each set of design variables defines a new cutout shape, which is generated and passed to Abaqus for another analysis. The optimization loops continue to run until the maximum number of iterations allowed is reached. The optimization iteration runs until the convergence criterion is satisfied:

$$
\left|K_{B}^{i}-K_{B}^{i-1}\right| \leq 10^{-6}
$$

where $i$ is the current iteration counter.

\section{Results}

The optimized shape of the cutout and the bending stiffness $K_{B}$ at each iteration of the local optimization iteration are shown in Fig. 16. The contour plot of $F I_{1}$ in Fig. 16a shows that the optimized cutout fully satisfies the failure constraint. It is interesting to note that the optimized cutout shape in Fig. 16 is nonsymmetric. Additional shape optimization results for a single cutout have been presented in [36].

\section{Summary and Comparison of Results}

A general formulation for the design of cutouts in deployable thin shells has been developed, and it has been applied to a detailed study of a deployable corner joint. Three different approaches have been presented. The first approach was to carry out a finite element simulation of the folding of a shell without any cutouts, and then remove the regions of the shell that had been found to be most highly stressed in the folded configuration. This approach was based on the conjecture that "drilling out" this material would remove the stress peaks. However, it was found that the insertion of the cutout changed the behavior of the shell to such an extent that high stresses appeared elsewhere, and it was concluded that this approach does not work. The second approach was to formulate the topology optimization of the cutouts using level-set functions, which allowed a systematic evaluation of a wide range of designs by varying only a small number of geometric parameters. The third approach was to insert a single cutout and optimize its shape by searching for the global minimum of the design space defined by a set of control points. Both the second and third approaches have been successful and, interestingly, have provided different solutions with almost identical performance.

The deployed bending stiffness of the shells and the maximum value of the first failure index for all the designs obtained in the paper are summarized in Table $\underline{3}$ and are also visually depicted in Fig. 17.
Table 3 Summary of $K_{B}$ versus $\max F I_{1}$ for shell designs studied in the paper

\begin{tabular}{lccc}
\hline \hline Design & Name & $\max \left(F I_{1}\right)$ & $K_{B}, \mathrm{~N} \cdot \mathrm{m}$ \\
\hline Preliminary & $A_{1}$ & 7.06 & 5.33 \\
& $A_{2}$ & 4.47 & 2.02 \\
& $A_{3}$ & 8.64 & 1.79 \\
& $A_{4}$ & 10.35 & 1.53 \\
& $A_{5}$ & 0.99 & 1.05 \\
Optimized & $B_{1}$ & 0.95 & 2.06 \\
& $B_{2}$ & 0.85 & 2.08 \\
& $B_{3}$ & 0.98 & 1.90 \\
& $B_{4}$ & 0.53 & 1.44 \\
\hline \hline
\end{tabular}

The green-shaded region of this plot corresponds to designs with a safe value of the failure index. A most desirable design would lie near the upper-left corner of the plot, representing designs with a bending stiffness of around $5 \mathrm{~N} \cdot \mathrm{m}$, i.e., as high as the shell without any cutouts but without any damage from folding. This performance is not achievable. The figure shows that five of the nine proposed designs lie in the safe region of the plot, and the highest achievable stiffness is around $2 \mathrm{~N} \cdot \mathrm{m}$. Among these five designs, three are symmetric and two are not.

A shell without any cutouts, $A_{1}$, has been included in the set of designs as a baseline, and it is surprising that two joint designs with cutouts, $A_{3}$ and $A_{4}$, reach even higher values of the failure index than the baseline. Placing a small circular cutout at the center of the $A_{1}$ design produced design $A_{2}$, which has less than half the bending stiffness and a lower value of the failure index, but still much larger than one. These results demonstrate the complex and counterintuitive variation of the maximum stress in a shell when cutouts are inserted.

Two more designs with a single cutout, $A_{3}$ and $A_{4}$, have a bending stiffness lower than $2.0 \mathrm{~N} \cdot \mathrm{m}$ and a maximum first failure index higher than 8 . These particular designs have sharp corners, and it is likely that a lower value of the maximum failure index would be obtained by rounding the transition between the shell. Design $A_{5}$, with a smooth transition between the two cylinders and a very large cutout that fully removes the two-play laminate region, leaves in place only two curved tape springs connecting the two parts of the joint. It satisfies the failure constraint but suffers a substantial penalty in terms of the bending stiffness. Overall, it is clear that the $A_{i}$ designs, generated without a formal optimization, were either unsuccessful or performed rather poorly.

The $B_{i}$ designs, obtained from topology or shape optimization, were superior to the $A_{i}$ designs. Design $B_{1}$ uses a single cutout with nonsymmetric shape and concurrently satisfies the failure constraint and maximizes the overall bending stiffness of the deployed shell. It

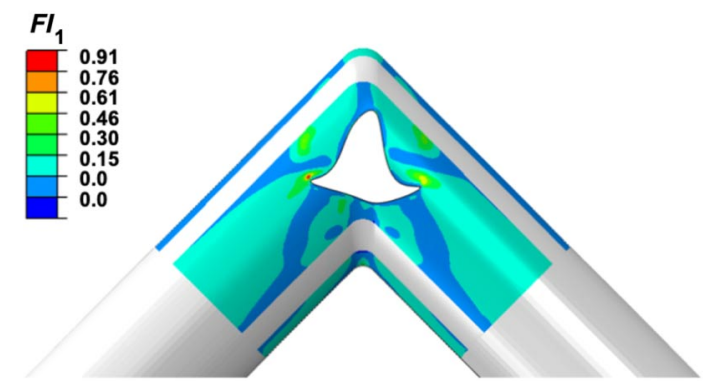

a) $F I_{1}$ mapped on deployed shell

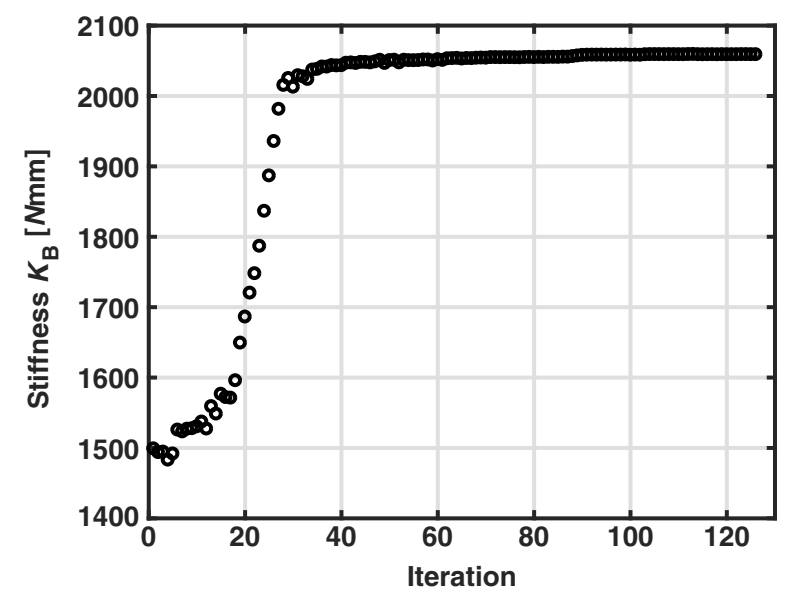

b) Evolution of objective function

Fig. 16 Shape optimization of a single cutout. 


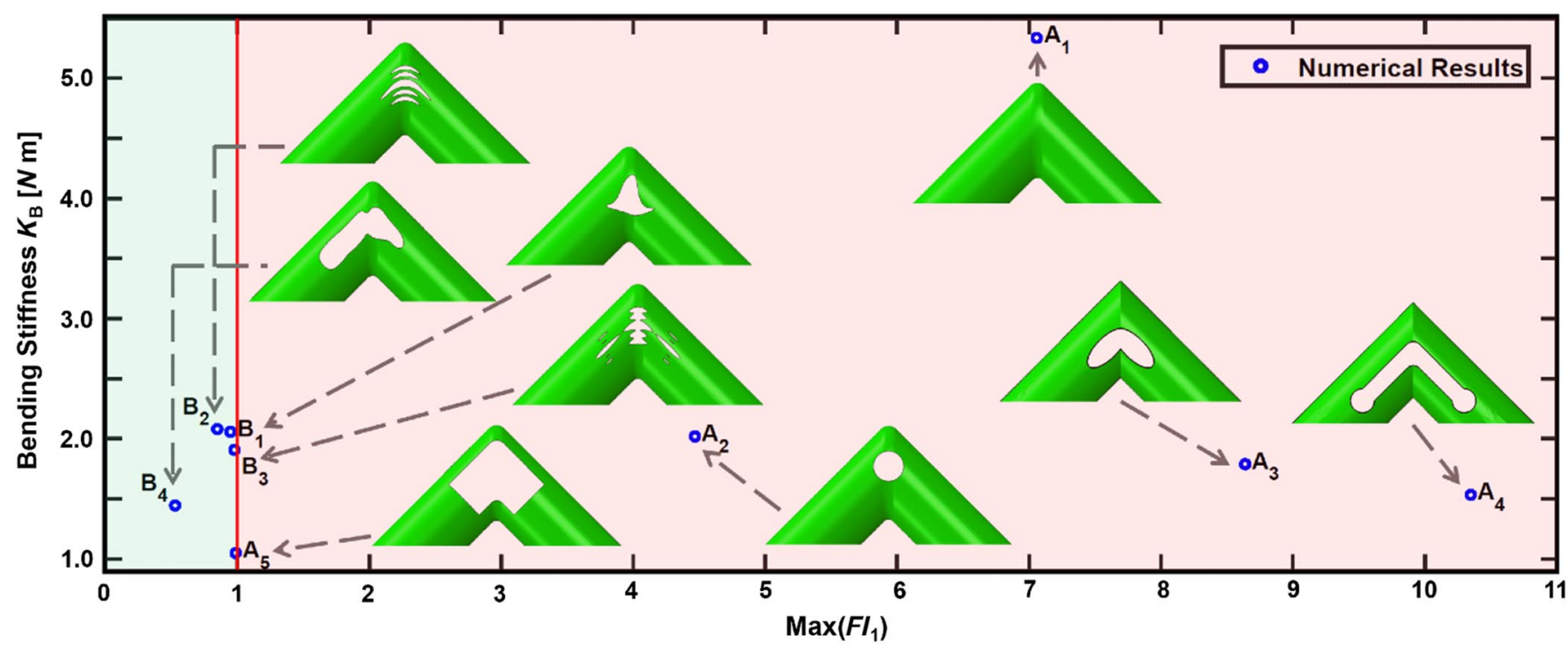

Fig. $17 K_{B}$ versus max $F I_{1}$ for shell designs studied in the paper.

is the second-best performer overall. Three designs obtained from topology optimization, $B_{2}, B_{3}$, and $B_{4}$, are shown in Fig. 17. Among these, design $B_{2}$ provides the highest bending stiffness while keeping the maximum failure index below 1. A physical model of this design was built and tested (Sec. IV.A.3) and showed no signs of damage after being folded.

All of the studies presented in this paper were carried out with the Abaqus commercial finite element software. Publicly available optimization software, combined with Python scripts that run Abaqus, were used to implement the optimization algorithms and to evaluate the failure indices. Sample codes are available at the following link: https://github.com/sergio-pellegrino/Serena-files.

\section{Conclusions}

It has been shown that thin shells with cutouts have a significant potential for deployable structures applications, and that topology/ shape optimization is an essential tool to design structures that achieve the best possible performance. An unexpected result of the study is that the best two deployable joint designs (joint $B_{1}$ with a single cutout and joint $B_{2}$ with five cutouts) are different in geometry but practically equivalent in terms of the chosen performance metrics. This results suggests that the ultimate performance for the chosen material and global shell geometry may have been reached.

An interesting observation is that one of these designs is symmetric and the other design is not, which leads to the question of what is the role of symmetry in the design of cutouts. Considering the folded shapes that have been previously seen in booms with cutouts [2, 4] $]$, it is not surprising that a nonsymmetric cutout has been obtained from the shape optimization. However, finding a symmetric design with equivalent performance is indeed surprising. It would be worth studying the design space near these two cutout designs, both with and without symmetry constraints, to better understand the role of symmetry breaking in shell folding.

Regarding the number of cutouts, shape optimization for two or three cutouts was also investigated during the course of the present study, but the results were inconclusive and have not been included in the paper. Separate cutouts have a tendency to merge into larger ones, and hence an algorithm that avoids merging of separate cutouts would need to be developed. Also, the computational burden of fully
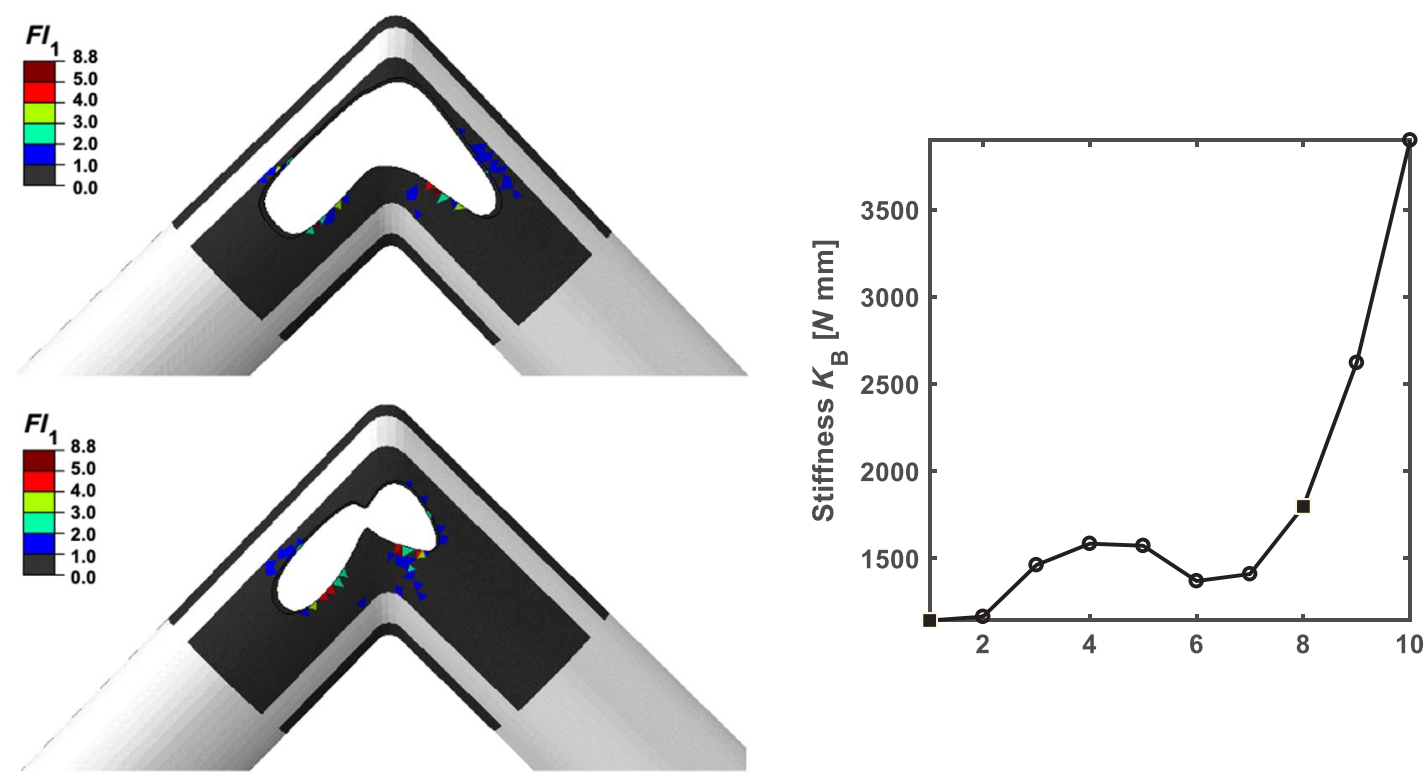

a) Cutouts corresponding to $z=0.1$ and $z=0.8$

b) Variation of bending stiffness for cutouts corresponding to $z=0.1,0.2, \ldots 1.0$

Fig. A1 Local minimum in the stiffness variation. 
investigating the shape optimization of multiple cutouts is significant. It had been conjectured that topology optimization and shape optimization would converge to similar cutout designs, but this conjecture could not be verified. This is an interesting topic for further research.

A further topic of future research is the extension of the range of folding angles to include the regime in which self-contact develops between different parts of the shell. It is believed that the cutout designs obtained in the present study would not change much, but it is likely that the second and third failure index would begin to play a greater role when self-contact forces the shell to reach higher curvature changes.

Additional topics that could be addressed in future work include further experimental verification to quantify the effects of randomly distributed manufacturing imperfections and the consideration of additional load conditions, instead of bending only, when optimizing the stiffness of the joints. For example, twisting or combined bending and twisting deformation modes could be considered to obtain different cutouts.

Finally, an important topic that has not been addressed in the present study is a detailed analysis of localized damage during folding of shells with cutouts. Some results of the present study show cutouts positioned very closely and forming thin strips of material that are to a certain extent softer and weaker than the rest of the shell, due to size effects. This topic has been addressed in a separate paper [37] and it has been shown that the results presented here are still valid.

\section{Appendix: Convexity of Objective Function}

The convexity of the function in Eq. (11) was investigated by computing $K_{B}$ for different types of variations of the cutout shape. An example in which monotonically varying level-set functions were considered is shown in Fig. A1. Figure A1 a shows two cutout shapes defined by Eq. (13) with $N_{h}=1, N_{l}=0$, intersected with cutting planes defined by $z=0.1$ and $z=0.8$. Figure A1b shows a plot of the stiffness $K_{B}$ for equally spaced cutting planes in the range $0.1-$ 1.0. This figure shows the existence of a local minimum for the particular type of cutout variation that has been considered.

Although it cannot be excluded that this minimum is in fact a saddle point, as it would require an investigation of the variation of $K_{B}$ for fully general variations of the cutout to reach a definite conclusion, this result indicates that the existence of local minima for $K_{B}$ is very likely. For this reason it is believed that the optimization problem is nonconvex.

\section{Acknowledgments}

This research was supported by a NASA Space Technology Research Fellowship. S.F. acknowledges the mentorship of William Doggett, NASA Langley Research Center, and valuable discussions with Thibaud Talon, Caltech. Suggestions from anonymous reviewers are gratefully acknowledged.

\section{References}

[1] Mobrem, M., and Adams, D. S., "Lenticular Jointed Antenna Deployment Anomaly and Resolution Onboard the Mars Express Spacecraft," Journal of Spacecraft and Rockets, Vol. 46, No. 2, 2009, pp. 403-410. https://doi.org/10.2514/1.36890

[2] Mallikarachchi, H. M. Y. C., and Pellegrino, S., "Quasi-Static Folding and Deployment of Ultrathin Composite Tape-Spring Hinges," Journal of Spacecraft and Rockets, Vol. 48, No. 1, 2011, pp. 187-198. https://doi.org/10.2514/1.47321

[3] Tan, L. T., and Pellegrino, S., "Thin-Shell Deployable Reflectors with Collapsible Stiffeners Part 1: Approach," AIAA Journal, Vol. 44, No. 11, 2006, pp. 2515-2523. https://doi.org/10.2514/1.16320

[4] Mallikarachchi, H. M. Y. C., and Pellegrino, S., "Optimized Designs of Composite Booms with Tape Spring Hinges," 51 st AIAA/ASME/ASCE/ AHS/ASC Structures, Structural Dynamics, and Materials Conference, AIAA Paper 2010-2750, 2010.
[5] Bendsøe, M. P., and Kikuchi, N., "Generating Optimal Topologies in Structural Design Using a Homogenization Method," Computer Methods in Applied Mechanics and Engineering, Vol. 71, No. 2, 1988, pp. 197-224. https://doi.org/10.1016/0045-7825(88)90086-2

[6] Osher, S., and Sethian, J. A., "Fronts Propagating with CurvatureDependent Speed: Algorithms Based on Hamilton-Jacobi Formulations," Journal of Computational Physics, Vol. 79, No. 1, 1988, pp. $12-49$. https://doi.org/10.1016/0021-9991(88)90002-2

[7] Sigmund, O., and Maute, K., "Topology Optimization Approaches," Structural and Multidisciplinary Optimization, Vol. 48, No. 6, 2013, pp. 1031-1055. https://doi.org/10.1007/s00158-013-0978-6

[8] Ye, Q., Guo, Y., Chen, S., Lei, N., and Gu, X. D., "Topology Optimization of Conformal Structures on Manifolds Using Extended Level Set Methods (X-LSM) and Conformal Geometry Theory," Computer Methods in Applied Mechanics and Engineering, Vol. 344, Feb. 2019, pp. 164-185. https://doi.org/10.1016/j.cma.2018.08.045

[9] Maute, K., Tkachuk, A., Wu, J., Qi, H. J., Ding, Z., and Dunn, M. L., "Level Set Topology Optimization of Printed Active Composites," Journal of Mechanical Design, Vol. 137, No. 11, 2015, Paper 111402. https://doi.org/10.1115/1.4030994

[10] Geiss, M. J., Boddeti, N., Weeger, O., Maute, K., and Dunn, M. L., "Combined Level-Set-XFEM-Density Topology Optimization of FourDimensional Printed Structures Undergoing Large Deformation," Journal of Mechanical Design, Vol. 141, No. 5, 2019, Paper 051405. https://doi.org/10.1115/1.4041945

[11] Ferraro, S., "Topology Optimization and Failure Analysis of Deployable Thin Shells with Cutouts," Ph.D. Dissertation, California Inst. of Technology, Pasadena, CA, 2020.

[12] JPS Technical Reference Handbook, JPS Composite Materials, Anderson, SC, 2017.

[13] Patz, M. T., PMT-F6 Toughened Cyanate Ester Resin System, Benicia, CA, 2019.

[14] Soykasap, O., "Deployment Analysis of a Self-Deployable Composite Boom," Composite Structures, Vol. 89, No. 3, 2009, pp. 374-381. https://doi.org/10.1016/j.compstruct.2008.08.012

[15] Banik, J., and Murphey, T., "Performance Validation of the Triangular Rollable and Collapsible Mast," 24th Annual AIAA/USU Conference on Small Satellites, 2010.

[16] Stabile, A., and Laurenzi, S., "Coiling Dynamic Analysis of ThinWalled Composite Deployable Boom," Composite Structures, Vol. 113, July 2014, pp. 429-436. https://doi.org/10.1016/j.compstruct.2014.03.043

[17] Peterson, L., and Mobrem, M., "Structural Analysis Methodology for Space Deployable Structures Using a High Performance Parallel Nonlinear Finite Element Solver," 4th AIAA Spacecraft Structures Conference, AIAA Paper 2017-0852, 2017. https://doi.org/10.2514/6.2017-0852

[18] Mobrem, M., Peterson, L., Cormarkovic, V., and Montazersadgh, F., "An Evaluation of Structural Analysis Methodologies for Space Deployable Structures," 4th AIAA Spacecraft Structures Conference, AIAA Paper 2017-0851, 2017. https://doi.org/10.2514/6.2017-0851

[19] Soykasap, O., "Micromechanical Models for Bending Behavior of Woven Composites," Journal of Spacecraft and Rockets, Vol. 43, No. 5, 2006, pp. 1093-1100. https://doi.org/10.2514/1.18010

[20] Mallikarachchi, H. M. Y. C., and Pellegrino, S., "Failure Criterion for Two-Ply Plain-Weave CFRP Laminates," Journal of Composite Materials, Vol. 47, No. 11, 2013, pp. 1357-1375. https://doi.org/10.1177/0021998312447208

[21] Fleck, N. A., and Budiansky, B., "Compressive Failure of Fibre Composites due to Microbuckling," Inelastic Deformation of Composite Materials, Springer, New York, 1991, pp. 235-273. https://doi.org/10.1007/978-1-4613-9109-8_12

[22] Yee, J. C. H., "Thin CFRP Composite Deployable Structures," Ph.D. Dissertation, Univ. of Cambridge, Cambridge, England, U.K., 2006.

[23] Yee, J. C. H., and Pellegrino, S., "Folding of Woven Composite Structures," Composites Part A: Applied Science and Manufacturing, Vol. 36, No. 2, 2005, pp. 273-278. https://doi.org/10.1016/S1359-835X(04)00167-8

[24] Murphey, T. W., Francis, W., Davis, B., and Mejia-Ariza, J. M., "High Strain Composites," 2nd AIAA Spacecraft Structures Conference, AIAA Paper 2015-0942, 2015. https://doi.org/10.2514/6.2015-0942

[25] Haftka, R. T., and Grandhi, R. V., "Structural Shape Optimization-A Survey," Computer Methods in Applied Mechanics and Engineering, 
Vol. 57, No. 1, 1986, pp. 91-106. https://doi.org/10.1016/0045-7825(86)90072-1

[26] Sethian, J. A., and Wiegmann, A., "Structural Boundary Design via Level Set and Immersed Interface Methods," Journal of Computational Physics, Vol. 163, No. 2, 2000, pp. 489-528. https://doi.org/10.1006/jcph.2000.6581

[27] Osher, S. J., and Santosa, F., "Level Set Methods for Optimization Problems Involving Geometry and Constraints: I. Frequencies of a Two-Density Inhomogeneous Drum," Journal of Computational Physics, Vol. 171, No. 1, 2001, pp. 272-288. https://doi.org/10.1006/jcph.2001.6789

[28] Wang, M. Y., Wang, X., and Guo, D., "A Level Set Method for Structural Topology Optimization," Computer Methods in Applied Mechanics and Engineering, Vol. 192, Nos. 1-2, 2003, pp. 227-246. https://doi.org/10.1016/S0045-7825(02)00559-5

[29] Allaire, G., Jouve, F., and Toader, A. M., "Structural Optimization Using Sensitivity Analysis and a Level-Set Method," Journal of Computational Physics, Vol. 194, No. 1, 2004, pp. 363-393. https://doi.org/10.1016/j.jcp.2003.09.032

[30] Ferraro, S., and Pellegrino, S., "Self-Deployable Joints for Ultra-Light Space Structures," SciTech 2018, AIAA Paper 2018-0694, 2018. https://doi.org/10.2514/6.2018-0694

[31] Royer, F., and Pellegrino, S., "Buckling of Ultralight Ladder-Type Coilable Space Structures," SciTech 2020, AIAA Paper 2020-1437, 2020. https://doi.org/10.2514/6.2020-1437

[32] Wales, D. J., and Doye, J. P., "Global Optimization by Basin-Hopping and the Lowest Energy Structures of Lennard-Jones Clusters Containing up to 110 Atoms," Journal of Physical Chemistry A, Vol. 101, No. 28 , 1997 , pp. 5111-5116.

https://doi.org/10.1021/jp970984n

[33] Powell, M. J., "A Direct Search Optimization Method that Models the Objective and Constraint Functions by Linear Interpolation," Advances in Optimization and Numerical Analysis, Springer, Dordrecht, 1994, pp. 51-67.

[34] Li, Z., and Scheraga, H. A., "Monte Carlo-Minimization Approach to the Multiple-Minima Problem in Protein Folding," Proceedings of the National Academy of Sciences, Vol. 84, No. 19, 1987, pp. 6611-6615.

https://doi.org/10.1073/pnas.84.19.6611.

[35] Perez, R. E., Jansen, P. W., and Martins, J. R. R. A., "pyOpt: A PythonBased Object-Oriented Framework for Nonlinear Constrained Optimization," Structural and Multidisciplinary Optimization, Vol. 45, No. 1, 2012, pp. 101-118.

https://doi.org/10.1007/s00158-011-0666-3

[36] Ferraro, S., and Pellegrino, S., "Topology Optimization of Composite Self-Deployable Shells with Cutouts," SciTech 2019, AIAA Paper 2019-1524, Jan. 2019.

https://doi.org/10.2514/6.2019-1524

[37] Ferraro, S., and Pellegrino, S., "Size Effects in Plain-Weave Astroquartz Deployable Thin Shells," Journal of Composite Materials, 2021. https://doi.org/10.1177/0021998320987618 